\title{
SUPG stabilization for the nonconforming virtual element method for advection-diffusion-reaction equations ${ }^{\text {th }}$
}

\author{
S. Berrone ${ }^{\mathrm{a}}$, A. Borio ${ }^{\mathrm{a}}$, G. Manzini ${ }^{\mathrm{b}, \mathrm{c}}$ \\ ${ }^{a}$ Dipartimento di Scienze Matematiche, Politecnico di Torino \\ Corso Duca degli Abruzzi 24, Torino, 10129, Italy \\ ${ }^{b}$ T-5 Group, Theoretical Division, Los Alamos National Laboratory, \\ Los Alamos, New Mexico, USA \\ ${ }^{c}$ Istituto di Matematica Applicata e Tecnologie Informatiche - CNR, \\ via Ferrata 1, Pavia 27100, Italy
}

\begin{abstract}
We present the design, convergence analysis and numerical investigations of the nonconforming virtual element method with Streamline Upwind/Petrov-Galerkin (VEM-SUPG) stabilization for the numerical resolution of convection-diffusion-reaction problems in the convective-dominated regime.

According to the virtual discretization approach, the bilinear form is split as the sum of a consistency and a stability term. The consistency term is given by substituting the functions of the virtual space and their gradients with their polynomial projection in each term of the bilinear form (including the SUPG stabilization term). Polynomial projections can be computed exactly from the degrees of freedom. The stability term is also built from the degrees of freedom by ensuring the correct scalability properties with respect to the mesh size and the equation coefficients.

The nonconforming formulation relaxes the continuity conditions at cell interfaces and a weaker regularity condition is considered involving polynomial moments of the solution jumps at cell interface. Optimal convergence properties of the method are proved in a suitable norm, which includes contribution from the advective stabilization terms. Experimental results confirm the theoretical convergence rates.
\end{abstract}

Keywords: Virtual Element Methods, Advection-diffusion-reaction problem, SUPG, stability, convergence

2000 MSC: 65N30, 65M12

\footnotetext{
G. Manzini has been partially supported by the Laboratory Directed Research and Development program (LDRD), U.S. Department of Energy Office of Science, Office of Fusion Energy Sciences, under the auspices of the National Nuclear Security Administration of the U.S. Department of Energy by Los Alamos National Laboratory, operated by Los Alamos National Security LLC under contract DE-AC5206NA25396. This work is assigned the LA-UR number LA-UR-17-20866. The three authors are also members of the Gruppo Nazionale Calcolo Scientifico (GNCS) at Istituto Nazionale di Alta Matematica (INdAM). S. Berrone and A. Borio kindly acknowledge partial financial support by INdAM-GNCS Projects 2018, HPC@Polito and by the MIUR project "Dipartimenti di Eccellenza 2018-2022".
} 


\section{Introduction}

The virtual element method (VEM) was proposed in [1] as a variational reformulation of the nodal mimetic finite difference (MFD) method 25 for solving diffusion problems on unstructured polygonal meshes. A survey on the MFD method can be found in the review paper [6] and the research book [7]. The VEM inherits the great flexibility of the MFD method with respect to the admissible meshes, and, despite its introduction dates back to a few years ago a huge amount of development has taken place, see, for example, [8 31. We emphasize that the VEM is not the only existing way to treat partial differential equations numerically on unstructured meshes. Other methods or families of methods that are available from the literature include the polygonal/polyhedral finite element method (PFEM) [32, 33], the BEM-based FEM [34, 35, the finite volume methods [36, 37, hybrid high-order (HHO) method 38, the discontinuous Galerkin (DG) method [39, 40, and the hybridized discontinuous Galerkin (HDG) method 41. Many of these methods are also part of the Gradient Scheme framework recently proposed by [42, 43. Moreover, the connection between VEM and finite elements on polygonal/polyhedral meshes is touroughly investigated in [44]46], between VEM and BEM-based FEM method in 47.

The virtual element method is a finite element method, but is dubbed virtual because its formulation does not require the explicit knowledge of a set of shape functions and gradients of shape functions to compute the bilinear forms, e.g., mass and stiffness matrices. The global approximation space is defined over the whole domain by gluing together local elemental spaces under some regularity constraint. Each elemental space is formed by the solutions of a local Poisson problem with a polynomial right-hand side and nonhomogeneous polynomial Dirichlet (or Neumann) boundary conditions. Clearly, a subspace of polynomials up to a given degree always belongs by construction to each elemental space. The remarkable fact is that we can compute exactly the projections of the virtual functions and their first derivatives onto such polynomials by using only the degrees of freedom. Therefore, a straightforward strategy to approximate the bilinear forms is to substitute the shape functions and their derivatives in their arguments with their polynomial projections. This approach yields the so-called consistency term, to which we add a stability term that ensures the nonsingularity of the resulting discretization. The stability term is designed to be easily computable from the degrees of freedom.

The VEM was originally formulated in [1] as a conforming FEM for the Poisson problem. It was later extended to convection-reaction-diffusion problems with variable coefficients in [12, 48. Meanwhile, the nonconforming formulation for diffusion problems was proposed in [49] as the finite element reformulation of 50, and later extended to general elliptic problems [51, Stokes problem [52, and the biharmonic equation [53, 54]. The two major differences between the conforming and nonconforming formulations are:

(i) at the elemental level the virtual space is formed by the solution of a Poisson problem with Neumann boundary conditions;

(ii) at the global level we relax the interelement conformity requirement, and the definition of the global discrete space just relays on some form of weaker regularity according to 55 . 
Nonconforming finite element spaces were historically proposed to approximate the velocity field of the Stokes equations on triangular meshes [55]. The functions in these finite element spaces are piecewise polynomials of degree $k=1$ [55, $k=2$ [56], $k=3$ [57, and $k>35860$. In such formulations, continuity is required only at a discrete set of special points located at cell interfaces, which are the roots of the one-dimensional $k^{\text {th }}$-order Legendre polynomials defined over each edge, i.e., the nodes of the GaussLegendre quadrature rule of order $k$. This minimal continuity requirement ensures the optimal convergence rate; see, for instance, [55. Attempts to extend non-conforming finite elements to quadrilaterals, tetrahedra and hexahedra are found in 61 63. A major issue of the nonconforming formulations is that they may strongly depend on the parity of the underlying polynomial space, the geometric shape of the element and its spatial dimensionality (2-D or 3-D). For example, on triangles the nonconforming finite element space for even $k \geq 2$ in [56, 58, 59] must be enriched by a one-dimensional subspace generated by a bubble function. Also, the definition of nonconforming spaces is substantially different from $2 \mathrm{D}$ and $3 \mathrm{D}$ and requires a simple geometric shape for the element (e.g., a simplex, a quadrilateral, or an hexahedral cell), and also differs from 2D to 3D. Instead, the nonconforming virtual element space proposed in [49] has the same construction for every $k$ regardless of the parity, the space dimension, and the elemental geometric shape.

In the case of the convection-dominated regime, a stabilization must be included in the variational formulation to deal with high Péclet number situations. In finite element approximations, different strategies have been designed to such purpose, as, for example, local projections [64, bubble functions [65, 66] the SUPG method [67-72]. The SUPG stabilization in the conforming virtual element formulation was previously considered in 73. The main goal of this work is the development of the nonconforming formulation with SUPG stabilization suitable to solve convection-dominated transport problems with a moderate reaction term. In such a situation the SUPG stabilization parameters becomes dependent on a local Péclet number and a local Karlovitz-like number. In case of a vanishing reaction the SUPG stabilization parameter converges to its expected classical definition. We prove the robustness of the method with respect to high Péclet numbers when the problem coefficients are constants and a conforming formulation is considered, whereas a weak dependence on the Péclet number is observed, due to the non-consistency of the VEM bilinear form [1] when the coefficients are variable or a non conforming formulation is considered. The presented analysis is also valid for SUPG-stabilized conforming virtual elements as presented in [73].

The outline of the paper is as follows. In Section 2 we introduce the mathematical model of the convection-reaction-diffusion problem. In Section 3 we present the nonconforming VEM with the SUPG stabilization for the convection-dominated regime. In Section 4 we carry out the convergence analysis and derive optimal a priori error estimates. In Section 5 we show the performance of the method on a set of representative problems. In Section 6 we offer our final remarks and conclusions.

\subsection{Notation}

The notation throughout the paper is as follows: $(\cdot, \cdot)$ and $\|\cdot\|$ denote the $\mathrm{L}^{2}(\Omega)$ scalar product and norm, and $(\cdot, \cdot)_{\omega}$ and $\|\cdot\|_{\omega}$ denote the $L^{2}(\omega)$ scalar product and norm defined on the subdomain $\omega \subseteq \Omega ;\|\cdot\|_{\alpha}$ and $|\cdot|_{\alpha}$ denote the $\mathrm{H}^{\alpha}(\Omega)$ norm and semi-norm; $\|\cdot\|_{\alpha, \omega}$ and $|\cdot|_{\alpha, \omega}$ denote the $\mathrm{H}^{\alpha}(\omega)$ norm and semi-norm; $\|\cdot\|_{\mathrm{W}_{p}^{q}(\omega)}$ and $|\cdot|_{\mathrm{W}_{p}^{q}(\omega)}$ denote the 
$\mathrm{W}_{p}^{q}(\omega)$ norm and semi-norm, where $p \geq 1$ is the Lebesgue regularity index and $q$ is the order of the Sobolev space. Moreover, $\mathbb{P}_{k}(\omega)$ denotes the space of polynomial functions of degree up to the integer number $k \geq 0$ that are defined on the $d$-dimensional subset $\omega \subseteq \Omega$ with $d=1,2,3$. If $\mathcal{T}_{h}$ is a partitioning of $\Omega$ in a set of non-overlapping polytopal elements $E$, i.e., the mesh, (for the formal definition see Section 3.1), by $\mathbb{P}_{k}\left(\mathcal{T}_{h}\right)$ we denote the space of discontinuous functions defined on $\Omega$ whose restriction to any element $E$ is a polynomial of degree less than or equal to $k$; hence, $p \in \mathbb{P}_{k}\left(\mathcal{T}_{h}\right)$ iff $p_{\mid E} \in \mathbb{P}_{k}(E)$. Finally, $\mathrm{H}^{t}\left(\mathcal{T}_{h}\right)$ for any $t \geq 1$ is the broken Sobolev space of globally $\mathrm{L}^{2}$-integrable functions on $\Omega$ whose restriction to any mesh element $E$ of the mesh $\mathcal{T}_{h}$ belongs to $\mathrm{H}^{t}(E)$; formally, we can write that

$$
\mathrm{H}^{t}\left(\mathcal{T}_{h}\right):=\left\{v \in \mathrm{L}^{2}(\Omega): v_{\mid E} \in \mathrm{H}^{t}(E), \forall E \in \mathcal{T}_{h}\right\} .
$$

To ease the notation, since these spaces contain discontinuous functions, in the following we will intend all norms and seminorms to be "broken" on the mesh. For example:

$$
\|\nabla v\|=\left(\sum_{E \in \mathcal{T}_{h}}\|\nabla v\|_{E}^{2}\right)^{\frac{1}{2}} .
$$

Furthermore, we will use the symbol $\mathcal{C}$ to denote a generic constant independent of the mesh size and the problem data $\mathrm{K}, \beta$ and $\gamma$. In the estimates this constant may have a different value for each occurrence.

\section{The variational formulation}

Let $\Omega \subset \mathbb{R}^{d}, d=2,3$ be a polytopal domain with boundary $\partial \Omega$ and consider the convection-diffusion-reaction problem:

$$
\begin{aligned}
-\nabla \cdot(\mathrm{K} \nabla u)+\beta \cdot \nabla u+\gamma u & =f & & \text { in } \Omega, \\
u & =0 & & \text { on } \partial \Omega,
\end{aligned}
$$

We assume that $\mathrm{K} \in\left[\mathrm{L}^{\infty}(\Omega)\right]^{d \times d}$ is a strongly elliptic and symmetric tensor almost everywhere (a.e.) on $\Omega$. Hence, there exist two positive constant $\kappa_{*}$ and $\kappa^{*}$ such that $\kappa_{*} \boldsymbol{\xi} \cdot \boldsymbol{\xi} \leq \boldsymbol{\xi} \cdot \mathrm{K}(x) \boldsymbol{\xi} \leq \kappa^{*} \boldsymbol{\xi} \cdot \boldsymbol{\xi}$ for every $\boldsymbol{\xi} \in \mathbb{R}^{d}$ and almost every $x \in \Omega$. We denote $\mathcal{C}_{\kappa}=\kappa^{*} / \kappa_{*}$. Moreover, we assume that $\beta \in\left[\mathrm{L}^{\infty}(\Omega)\right]^{d}$ with $\nabla \cdot \beta=0$ and $\gamma \in \mathrm{L}^{\infty}(\Omega)$ such that $\inf _{x \in \Omega} \gamma(x)=\gamma_{0} \geq 0$. To ease the exposition, we present the virtual element formulation and the convergence analysis assuming homogeneous Dirichlet boundary conditions. However, all the results presented in this paper can readily be extended to more general situations.

Consider the bilinear form $B: \mathrm{H}_{0}^{1}(\Omega) \times \mathrm{H}_{0}^{1}(\Omega) \rightarrow \mathbb{R}$ defined by

$$
B(w, v):=(\mathrm{K} \nabla w, \nabla v)+(\beta \cdot \nabla w, v)+(\gamma w, v) \quad \forall w, v \in \mathrm{H}_{0}^{1}(\Omega),
$$

and the linear functional $F: \mathrm{H}_{0}^{1}(\Omega) \rightarrow \mathbb{R}$ defined by

$$
F(v):=(f, v) \quad \forall v \in \mathrm{H}_{0}^{1}(\Omega) .
$$


The variational formulation of $(2)$ reads as: Find $u \in \mathrm{H}_{0}^{1}(\Omega)$ such that

$$
B(u, v)=F(v) \quad \forall v \in \mathrm{H}_{0}^{1}(\Omega) .
$$

The bilinear form $B$ is coercive and bounded, and the variational problem (4) has a unique solution in view of the Lax-Milgram lemma.

\section{The virtual element formulation}

Hereafter, we consider only the case for $d=2$. However, the nonconforming virtual element formulation is almost the same for $d=2$ and 3 , the main substantial difference being necessarily in the mesh assumptions that for $d=3$ must also consider a star-shaped condition on the faces. Therefore, most of the results presented in the next sections can easily be generalized to the three-dimensional case with minor or no changes at all.

\subsection{General assumptions}

Let $\left\{\mathcal{T}_{h}\right\}_{h}$ be a sequence of meshes of $\Omega$, i.e., a sequence of non-overlapping polygonal partitions of the domain $\Omega$. Each $\mathcal{T}_{h}$ is labeled by the subscript $h$, the maximum diameter of its polygonal elements $E$. The polygonal elements can have a different number of edges and hanging node-like configurations are possible with nodes placed on an edge and forming a flat angle. We denote the set of all the mesh edges $e$ of the polygonal cells in $\mathcal{T}_{h}$ by $\mathcal{E}_{h}$. We also distinguish between the subset of internal edges $\mathcal{E}_{h}^{\text {int }}$ and the subset of the boundary edges $\mathcal{E}_{h}^{b n d}$; clearly, $\mathcal{E}_{h}=\mathcal{E}_{h}^{\text {int }} \cup \mathcal{E}_{h}^{b n d}$.

We assume that the members of the sequence $\left\{\mathcal{T}_{h}\right\}_{h}$ satisfy the following regularity assumptions: There exists a global constant $\rho>0$ such that for each mesh $\mathcal{T}_{h}$ :

(i) every polygon $E \in \mathcal{T}_{h}$ is star-shaped with respect to a ball whose radius is greater than or equal to $\rho h_{E}$, where $h_{E}=\max _{\mathbf{x}, \mathbf{y} \in E}\|\mathbf{x}-\mathbf{y}\|$ is the element diameter;

(ii) $\forall E \in \mathcal{T}_{h}$, each side $e$ of $E$ is such that $h_{e} \geq \rho h_{E}$, where $h_{e}$ is the length of $e$;

Remark 1. Assumption (i) implies that each element is simply connected. Assumption (ii) implies that the number of sides of each polygon of the mesh is uniformly bounded over the mesh sequence.

The restriction of $\mathrm{K}$ to any element $E \in \mathcal{T}_{h}$ is still a strongly elliptic tensor and its spectrum can be locally bounded by using two constants $\mathrm{K}_{E}^{\vee}$ and $\mathrm{K}_{E}$, so that for any vector-valued field $\boldsymbol{\xi}(x)$ defined on $E$ it holds that

$$
\mathrm{K}_{E}^{\vee} \boldsymbol{\xi}(x) \cdot \boldsymbol{\xi}(x) \leq \boldsymbol{\xi}(x) \cdot \mathrm{K}(x) \boldsymbol{\xi}(x) \leq \mathrm{K}_{E} \boldsymbol{\xi}(x) \cdot \boldsymbol{\xi}(x) \quad \forall x \in E .
$$

We will find convenient for the next theoretical developments to assume that the inequalities $0<\kappa_{*} \leq \mathrm{K}_{E}^{\vee} \leq \mathrm{K}_{E} \leq \kappa^{*}$ holds true for every mesh element $E$. Since $\mathrm{K}$ is represented by a symmetric and positive definite matrix we consider the decomposition $\mathrm{K}=(\sqrt{\mathrm{K}})^{\top} \sqrt{\mathrm{K}}$ and we write $(\mathrm{K} \nabla v, \nabla v)_{E}=(\sqrt{\mathrm{K}} \nabla v, \sqrt{\mathrm{K}} \nabla v)_{E}=\|\sqrt{\mathrm{K}} \nabla v\|_{E}^{2}$ for any sufficiently regular function $v$. Therefore, setting $\boldsymbol{\xi}=\nabla v$ in (5) yields

$$
\mathrm{K}_{E}^{\vee}\|\nabla v\|_{E}^{2} \leq\|\sqrt{\mathrm{K}} \nabla v\|_{E}^{2} \leq \mathrm{K}_{E}\|\nabla v\|_{E}^{2}
$$


We will use this relation extensively in the analysis of the next sections. For each element $E \in \mathcal{T}_{h}$ we also set

$$
\beta_{E}:=\sup _{x \in E}\|\beta(x)\|_{\mathbb{R}^{2}}, \quad \gamma_{E}:=\|\gamma\|_{\infty, E} .
$$

Let $k \geq 0$ be an integer number and $\boldsymbol{\alpha}=\left(\alpha_{1}, \alpha_{2}\right)$ a two-dimensional multi-index of order $|\boldsymbol{\alpha}|=\alpha_{1}+\alpha_{2} \leq k$. The polynomial space $\mathbb{P}_{k}(E)$ is spanned by the monomials $m_{\boldsymbol{\alpha}} \in \mathcal{M}_{k}(E)$ defined as

$$
m_{\boldsymbol{\alpha}}(\mathbf{x}):=\frac{\left(\mathbf{x}-\mathbf{x}_{E}\right)^{\boldsymbol{\alpha}}}{h_{E}^{|\boldsymbol{\alpha}|}} \quad \forall \mathbf{x} \in E,
$$

where $\mathbf{x}_{E}$ is the center of the ball with respect to which $E$ is star-shaped. Similarly, $\mathbb{P}_{k}(e)$, the space of polynomials of degree $k$ defined on edge $e$, is spanned by the monomials $m_{\alpha}(\xi):=\left(\xi-\xi_{e}\right)^{\alpha} / h_{e}^{\alpha} \in \mathcal{M}_{k}(e)$ for $0 \leq \alpha \leq k$, where $\xi$ is a local coordinate defined on $e, \xi_{e}$ the midpoint of $e$, and $h_{e}$ the length of $e$.

In the formulation of the method we will make use of the elliptic projection operator $\Pi_{k}^{\nabla}: \mathrm{H}^{1}\left(\mathcal{T}_{h}\right) \rightarrow \mathbb{P}_{k}\left(\mathcal{T}_{h}\right)$, whose restriction to each element $E$ is the solution of the local problem:

$$
\begin{cases}\left(\nabla \Pi_{k}^{\nabla} v, \nabla p\right)_{E}=(\nabla v, \nabla p)_{E} & \forall p \in \mathbb{P}_{k}(E) \\ \left(\Pi_{k}^{\nabla} v, 1\right)_{\partial E}=(v, 1)_{\partial E} & \text { if } k=1, \\ \left(\Pi_{k}^{\nabla} v, 1\right)_{E}=(v, 1)_{E} & \text { if } k>1 .\end{cases}
$$

We will also consider the $\mathrm{L}^{2}$-projection operator $\Pi_{l}^{0}: \mathrm{H}^{1}\left(\mathcal{T}_{h}\right) \rightarrow \mathbb{P}_{l}\left(\mathcal{T}_{h}\right)$ whose restriction to each element $E$ is the $\mathrm{L}^{2}$-projection onto $\mathbb{P}_{l}(E)$. A crucial property of these projection operators, which will be discussed in the next section (see Remark 2), is that they are computable on the functions of the virtual element space using only their degrees of freedom.

\subsection{The local nonconforming virtual element space}

The local nonconforming virtual element space of order $k \geq 1$ is defined as follows:

$$
\begin{aligned}
V_{h}^{E}:=\left\{v_{h} \in \mathrm{H}^{1}(E): \Delta v_{h} \in \mathbb{P}_{k}(E), \frac{\partial v_{h}}{\partial \hat{n}_{e}} \in \mathbb{P}_{k-1}(e) \forall e \subset \partial E,\right. \\
\left.\left(v_{h}, p\right)_{E}=\left(\Pi_{k}^{\nabla} v_{h}, p\right)_{E} \forall p \in \mathbb{P}_{k}(E) / \mathbb{P}_{k-2}(E)\right\},
\end{aligned}
$$

where $\mathbb{P}_{k}(E) / \mathbb{P}_{k-2}(E)$ is the subspace of $\mathbb{P}_{k}(E)$ of the polynomials that are $\mathrm{L}^{2}$-orthogonal to $\mathbb{P}_{k-2}(E)$ (or, alternatively, the polynomials whose degree is exactly $k-1$ and $k$ ), and for $k=1$ we conventionally take $\mathbb{P}_{-1}(E)=\{0\}$. The definition of $V_{h}^{E}$ is based on the enhancement strategy that was introduced in 48 for the conforming case and extended to the nonconforming case in [51]. From the definition above it follows immediately that $\mathbb{P}_{k}(E)$ is a linear subspace of $V_{h}^{E}$.

A function $v_{h} \in V_{h}^{E}$ is uniquely identified by the following set of degrees of freedom: 


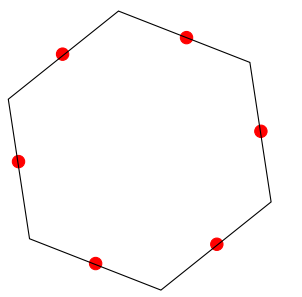

$\mathbf{k}=\mathbf{1}$

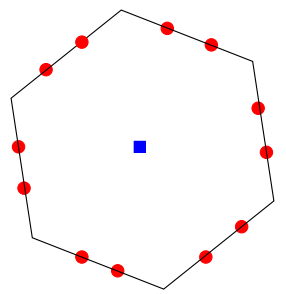

$\mathbf{k}=\mathbf{2}$

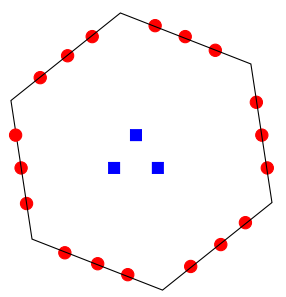

$\mathbf{k}=\mathbf{3}$

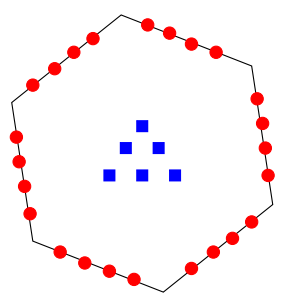

$\mathrm{k}=4$

Figure 1: Degrees of freedom of a hexagonal cell for $k=1,2,3$, 4; edge moments are marked by a circle; cell moments are marked by a square.

- for $k \geq 1$, the moments of $v_{h}$ of order up to $k-1$ on each mesh interface $e$ :

$$
\frac{1}{|e|} \int_{e} v_{h} m_{\alpha} d \xi \quad \forall m_{\alpha} \in \mathcal{M}_{k-1}(e) ;
$$

- for $k>1$, the moments of $v_{h}$ of order up to $k-2$ inside element $E$ :

$$
\frac{1}{|E|} \int_{E} v_{h} m_{\alpha} d \mathbf{x} \quad \forall m_{\alpha} \in \mathcal{M}_{k-2}(E),
$$

The unisolvency of these degrees of freedom is proved in 49. A counting argument shows that the cardinality of this set of degrees of freedom, which is also the dimension of $V_{h}^{E}$, is equal to $n_{E}(k-1)+k(k-1) / 2$, where $n_{E}$ is the number of edges of $E$. The degrees of freedom for an hexagonal cell are shown in Figure 1.

Remark 2. The elliptic projection $\Pi_{k}^{\nabla} v_{h}$ is computable from the degrees of freedom of $v_{h}$. In fact, an integration by parts of the right-hand side of (6) yields:

$$
\left(\nabla v_{h}, \nabla p\right)_{E}=-\left(v_{h}, \Delta p\right)_{E}+\sum_{e \in \partial E}\left(v_{h}, \mathbf{n}_{e} \cdot \nabla p\right)_{e}
$$

The terms on the right can be expressed by using the $(k-2)$-order moments of $v_{h}$ inside $E$ and the $(k-1)$-order moments of $v_{h}$ on each edge $e \in \partial E$ and are thus computable. A similar argument shows that also $\Pi_{k-1}^{0} v_{h}$ and $\Pi_{k-1}^{0} \nabla v_{h}$ are computable from the degrees of freedom of $v_{h}$.

\subsection{Global nonconforming virtual element spaces}

For the construction of the global virtual element spaces we introduce the nonconforming functional space

$$
\mathrm{H}_{k}^{1, n c}\left(\mathcal{T}_{h}\right):=\left\{v \in \mathrm{H}^{1}\left(\mathcal{T}_{h}\right): \int_{e} \llbracket v \rrbracket q d \xi=0 \quad \forall q \in \mathbb{P}_{k-1}(e) \forall e \in \mathcal{E}_{h}\right\},
$$

where $\llbracket \cdot \rrbracket$ denotes the jump operator $\llbracket \cdot \rrbracket$ across a mesh interface, which is defined as follows. If $e$ is an internal edge, we fix a unique unit normal vector $\hat{n}_{e}$ and we set $\llbracket v \rrbracket:=v^{+}-v^{-}$, where $v^{ \pm}$are the traces of $v$ on $e$ from within the two elements $E^{ \pm}$ 
sharing the edge, being $E^{+}$the element for which $\hat{n}_{e}$ is pointing outward. If $e$ is a boundary edge, $\hat{n}_{e}$ is orthogonal to $e$ and pointing out of the computational domain $\Omega$ and $\llbracket v \rrbracket:=v^{+}$.

Finally, the global nonconforming virtual element space of order $k$ is defined by

$$
V_{h}:=\left\{v_{h} \in \mathrm{H}_{k}^{1, n c}\left(\mathcal{T}_{h}\right): v_{h \mid E} \in V_{h}^{E} \quad \forall E \in \mathcal{T}_{h}\right\} .
$$

Each function $v_{h}$ of $V_{h}$ is uniquely characterized by:

- for $k \geq 1$, the moments of order up to $k-1$ on each internal mesh edge $e \in \mathcal{E}_{h}^{\text {int }}$ :

$$
\frac{1}{|e|} \int_{e} v_{h} m_{\alpha} d \xi \quad \forall m_{\alpha} \in \mathcal{M}_{k-1}(e)
$$

- for $k>1$, the moments of order up to $k-2$ inside each element $E \in \mathcal{T}_{h}$ :

$$
\frac{1}{|E|} \int_{E} v_{h} m_{\alpha} d \mathbf{x} \quad \forall m_{\alpha} \in \mathcal{M}_{k-2}(E) .
$$

The unisolvency of these degrees of freedom in $V_{h}$ is a direct consequence of the unisolvency of the local degrees of freedom introduced in section 3.2 and the definition of the nonconforming space $\mathrm{H}_{k}^{1, n c}\left(\mathcal{T}_{h}\right)$, cf. [49].

\subsection{SUPG-VEM formulation}

The discretization of the variational formulation (4) may lead to instabilities when the convective term $(\beta \cdot \nabla w, v)$ is dominant with respect to the diffusive term $(\mathrm{K} \nabla w, \nabla v)$. Here we consider also a moderate reaction term that we assume not to be source of instabilities. In this section we recast the classical Streamline Upwind Petrov Galerkin (SUPG) approach [68] in the framework of the nonconforming VEM, showing that the optimal order of convergence can be preserved. To this end, we assume that $\mathrm{K} \in\left[\mathrm{W}_{\infty}^{1}(\Omega)\right]^{d \times d}$. Then, we introduce the functional space

$$
V:=\left\{v \in \mathrm{H}_{0}^{1}(\Omega): \Delta v \in \mathrm{L}^{2}(E) \quad \forall E \in \mathcal{T}_{h}\right\},
$$

the bilinear form $B_{\text {supg }}: V \times \mathrm{H}_{0}^{1}(\Omega) \rightarrow \mathbb{R}$ given by

$$
B_{\text {supg }}(w, v):=a(w, v)+b(w, v)+c(w, v)+d(w, v),
$$

where

$$
\begin{aligned}
a(w, v) & :=\sum_{E \in \mathcal{T}_{h}}(\mathrm{~K} \nabla w, \nabla v)_{E}+\tau_{E}(\beta \cdot \nabla w, \beta \cdot \nabla v)_{E}, \\
b(w, v) & :=\frac{1}{2} \sum_{E \in \mathcal{T}_{h}}\left[(\beta \cdot \nabla w, v)_{E}-(w, \beta \cdot \nabla v)_{E}\right], \\
c(w, v) & :=\sum_{E \in \mathcal{T}_{h}}\left(\gamma w, v+\tau_{E} \beta \cdot \nabla v\right)_{E}, \\
d(w, v) & :=-\sum_{E \in \mathcal{T}_{h}} \tau_{E}(\nabla \cdot(\mathrm{K} \nabla w), \beta \cdot \nabla v)_{E} .
\end{aligned}
$$


Furthermore, let $F_{\text {supg }}: \mathrm{H}_{0}^{1}(\Omega) \rightarrow \mathbb{R}$ be the linear functional given by

$$
F_{\text {supg }}(v)=(f, v)+\sum_{E \in \mathcal{T}_{h}} \tau_{E}(f, \beta \cdot \nabla v)_{E} .
$$

The real positive factor $\tau_{E}$ is the local SUPG parameter and is discussed in section 3.5 . The SUPG variational formulation of problem (2) reads as: Find $u \in V$ such that

$$
B_{\text {supg }}(u, v)=F_{\text {supg }}(v) \quad \forall v \in \mathrm{H}_{0}^{1}(\Omega) .
$$

Remark 3. Under the assumptions of Section 2 , the bilinear term $b$ in (3) that corresponds to the convective flux is equivalent to the skew-symmetric term $b$ in (15).

Remark 4. By introducing the matrix $\mathrm{K}_{\beta, E}=\mathrm{K}+\tau_{E} \beta \beta^{\top}$, the bilinear form $a$ in (14) can be reformulated as:

$$
a(w, v):=\sum_{E \in \mathcal{T}_{h}} a^{E}(w, v)=\sum_{E \in \mathcal{T}_{h}}\left(\mathrm{~K}_{\beta, E} \nabla w, \nabla v\right)_{E} .
$$

Since matrix $\mathrm{K}_{\beta, E}$ is positive definite, we can use the decomposition $\mathrm{K}_{\beta, E}=\sqrt{\mathrm{K}_{\beta, E}} \sqrt{\mathrm{K}_{\beta, E}}$ and prove that the bilinear form is continuous, i.e,

$$
a^{E}(w, v) \leq\left\|\sqrt{\mathrm{K}_{\beta, E}} \nabla w\right\|_{E}\left\|\sqrt{\mathrm{K}_{\beta, E}} \nabla v\right\|_{E}
$$

which holds for every pair of nonconforming functions $v, w$.

The SUPG-stabilized virtual element approximation of (4) reads as: Find $u_{h} \in V_{h}$ such that

$$
B_{\text {supg }, h}\left(u_{h}, v_{h}\right)=F_{\text {supg }, h}\left(v_{h}\right) \quad \forall v_{h} \in V_{h},
$$

where the bilinear form $B_{\text {supg }, h}: V_{h} \times V_{h} \rightarrow \mathbb{R}$ and the right-hand side $F_{\text {supg, } h}: V_{h} \rightarrow \mathbb{R}$ are the virtual element approximation of $B_{\text {supg }}$ and $F_{\text {supg }}$, respectively. The bilinear form $B_{\text {supg }, h}$ is given by

$$
B_{\text {supg }, h}\left(w_{h}, v_{h}\right):=a_{h}\left(w_{h}, v_{h}\right)+b_{h}\left(w_{h}, v_{h}\right)+c_{h}\left(w_{h}, v_{h}\right)+d_{h}\left(w_{h}, v_{h}\right)
$$

for any $w_{h}, v_{h} \in V_{h}$, where

$$
\begin{aligned}
& a_{h}\left(w_{h}, v_{h}\right):=\sum_{E \in \mathcal{T}_{h}}\left(\left(\mathrm{~K} \Pi_{k-1}^{0} \nabla w_{h}, \Pi_{k-1}^{0} \nabla v_{h}\right)_{E}+\tau_{E}\left(\beta \cdot \Pi_{k-1}^{0} \nabla w_{h}, \beta \cdot \Pi_{k-1}^{0} \nabla v_{h}\right)_{E}\right. \\
& \left.+S^{E}\left(\left(I-\Pi_{k-1}^{0}\right) w_{h},\left(I-\Pi_{k-1}^{0}\right) v_{h}\right)\right), \\
& b_{h}\left(w_{h}, v_{h}\right):=\sum_{E \in \mathcal{T}_{h}} \frac{1}{2}\left(\left(\beta \cdot \Pi_{k-1}^{0} \nabla w_{h}, \Pi_{k-1}^{0} v_{h}\right)_{E}-\left(\Pi_{k-1}^{0} w_{h}, \beta \cdot \Pi_{k-1}^{0} \nabla v_{h}\right)_{E}\right), \\
& c_{h}\left(w_{h}, v_{h}\right):=\sum_{E \in \mathcal{T}_{h}}\left(\gamma \Pi_{k-1}^{0} w_{h}, \Pi_{k-1}^{0} v_{h}+\tau_{E} \beta \cdot \Pi_{k-1}^{0} \nabla v_{h}\right)_{E}, \\
& d_{h}\left(w_{h}, v_{h}\right):=-\sum_{E \in \mathcal{T}_{h}} \tau_{E}\left(\nabla \cdot\left(\mathrm{K} \Pi_{k-1}^{0} \nabla w_{h}\right), \beta \cdot \Pi_{k-1}^{0} \nabla v_{h}\right)_{E},
\end{aligned}
$$


the local VEM stabilization term in $a_{h}\left(w_{h}, v_{h}\right)$ is given by

$$
S^{E}\left(\left(I-\Pi_{k-1}^{0}\right) w_{h},\left(I-\Pi_{k-1}^{0}\right) v_{h}\right):=\left(\mathrm{K}_{E}+\tau_{E} \beta_{E}^{2}\right) S_{*}^{E}\left(\left(I-\Pi_{k-1}^{0}\right) w_{h},\left(I-\Pi_{k-1}^{0}\right) v_{h}\right),
$$

where $S_{*}^{E}\left(\left(I-\Pi_{k-1}^{0}\right) v_{h},\left(I-\Pi_{k-1}^{0}\right) v_{h}\right)$ is such that there exist two constants $\sigma^{*}, \sigma_{*}>$ 0 , independent of $h$ and the problem parameters satisfying, $\forall v_{h} \in V_{h}$,

$\sigma_{*}\left\|\nabla\left(I-\Pi_{k-1}^{0}\right) v_{h}\right\|_{E}^{2} \leq S_{*}^{E}\left(\left(I-\Pi_{k-1}^{0}\right) v_{h},\left(I-\Pi_{k-1}^{0}\right) v_{h}\right) \leq \sigma^{*}\left\|\nabla\left(I-\Pi_{k-1}^{0}\right) v_{h}\right\|_{E}^{2}$.

Moreover, the linear functional $F_{\mathrm{supg}, h}\left(v_{h}\right)$ is given by

$$
F_{\text {supg }, h}\left(v_{h}\right)=\left(f, \Pi_{k-1}^{0} v_{h}\right)+\sum_{E \in \mathcal{T}_{h}} \tau_{E}\left(f, \beta \cdot \Pi_{k-1}^{0} \nabla v_{h}\right)_{E},
$$

for any $v_{h} \in V_{h}$. In view of Remark 4 , we can define the local bilinear form

$$
a_{h}^{E}\left(w_{h}, v_{h}\right)=\left(\mathrm{K}_{\beta, E} \Pi_{k-1}^{0} \nabla w_{h}, \Pi_{k-1}^{0} \nabla v_{h}\right)_{E}
$$

such that

$$
a_{h}\left(w_{h}, v_{h}\right)=\sum_{E \in \mathcal{T}_{h}}\left(a_{h}^{E}\left(w_{h}, v_{h}\right)+S^{E}\left(\left(I-\Pi_{k-1}^{0}\right) w_{h},\left(I-\Pi_{k-1}^{0}\right) v_{h}\right)\right) .
$$

Notice that, by [21], 28] and (29), the stabilization term $S^{E}: V_{h} \times V_{h} \rightarrow \mathbb{R}$ satisfies

$$
\begin{aligned}
S^{E}\left(\left(I-\Pi_{k-1}^{0}\right) v_{h},\left(I-\Pi_{k-1}^{0}\right) v_{h}\right) & \geq \sigma_{*}\left(\mathrm{~K}_{E}+\tau_{E} \beta_{E}^{2}\right)\left\|\nabla v_{h}-\nabla \Pi_{k-1}^{0} v_{h}\right\|_{E}^{2} \\
& \geq \sigma_{*}\left(\mathrm{~K}_{E}+\tau_{E} \beta_{E}^{2}\right)\left\|\nabla v_{h}-\Pi_{k-1}^{0} \nabla v_{h}\right\|_{E}^{2} \\
& \geq \sigma_{*}\left\|\sqrt{\mathrm{K}_{\beta, E}}\left(\nabla v_{h}-\Pi_{k-1}^{0} \nabla v_{h}\right)\right\|_{E}^{2},
\end{aligned}
$$

being $\left\|\nabla v_{h}-\Pi_{k-1}^{0} \nabla v_{h}\right\|_{E} \leq\left\|\nabla v_{h}-\nabla \Pi_{k-1}^{0} v_{h}\right\|_{E}$. According to [51], a possible choice for $S_{*}^{E}$ is given by

$$
S_{*}^{E}\left(\left(I-\Pi_{k-1}^{0}\right) w_{h},\left(I-\Pi_{k-1}^{0}\right) v_{h}\right)=\sum_{i=1}^{N_{E}} \chi_{i}\left(\left(I-\Pi_{k-1}^{0}\right) w_{h}\right) \chi_{i}\left(\left(I-\Pi_{k-1}^{0}\right) v_{h}\right),
$$

where $N_{E}$ is the number of degrees of freedom on the element $E$ and $\chi_{i}$ is the operator that selects the $i$-th degree of freedom.

The effect of the SUPG stabilization in the VEM stabilization is reflected by the term $\tau_{E} \beta_{E}^{2}$ that appears in the local coefficient multiplying $S^{E}$ in definition 28].

\subsection{The $S U P G$ parameter $\tau_{E}$}

According to [68, 73], the stability parameter $\tau_{E}$ when there is no reaction term is defined by

$$
\tau_{E}=\frac{h_{E}}{2 \beta_{E}} \min \left\{\mathrm{Pe}_{E}, 1\right\}, \quad \text { where } \quad \mathrm{Pe}_{E}:=m_{k}^{E} \frac{\beta_{E} h_{E}}{2 \mathrm{~K}_{E}},
$$


and

$$
m_{k}^{E}:= \begin{cases}\frac{1}{3} & \text { if } \nabla \cdot\left(\mathrm{K} \nabla v_{h}\right)=0 \forall v_{h} \in V_{h}^{E}, \\ 2 \tilde{C}_{k}^{E} & \text { otherwise }\end{cases}
$$

is the mesh Péclet number of $E ; \tilde{C}_{k}^{E}$ is the biggest constant number satisfying the following inverse inequality:

$$
\tilde{C}_{k}^{E} h_{E}^{2}\left\|\nabla \cdot\left(\mathrm{K} \nabla v_{h}\right)\right\|_{E}^{2} \leq\left\|\mathrm{K} \nabla v_{h}\right\|_{E}^{2} \quad \forall v_{h} \in V_{h}^{E} .
$$

A proof of such a local inverse inequality for the virtual element space $V_{h}^{E}$ is provided in [9, Lemma 10] for constant $\mathrm{K}$ and any function with polynomial laplacian under the current mesh regularity assumptions. For a nonconstant K, using standard manipulations we obtain (34) with a constant $\tilde{C}_{k}^{E}$ that may depend on the variations of K on the element.

In [68, 73] no reaction term was considered. Since here we have such a term we need to modify the definition of $\tau_{E}$ by adding a constraint that guarantees the coercivity of $B_{\text {supg }}$ and $B_{\text {supg, } h}$. As in the proof of Lemma 1 we need that $\frac{1}{2}-\frac{\gamma_{E} \tau_{E}}{2} \geq C>0$, we may assume that there exists a constant $C_{\tau} \in(0,1)$ such that

$$
\tau_{E}:=\min \left\{\frac{\tilde{C}_{k}^{E} h_{E}^{2}}{\mathrm{~K}_{E}}, \frac{h_{E}}{2 \beta_{E}}, \frac{C_{\tau}}{\gamma_{E}}\right\} .
$$

Finally, we introduce the local Karlovitz number, i.e., the dimensionless parameter associated with each mesh element $E$,

$$
\mathrm{Ka}_{E}:=\frac{2 \beta_{E} C_{\tau}}{h_{E} \gamma_{E}},
$$

and we redefine $\tau_{E}$ as

$$
\tau_{E}=\frac{h_{E}}{2 \beta_{E}} \min \left\{\mathrm{Pe}_{E}, 1, \mathrm{Ka}_{E}\right\} .
$$

The comparison between $\mathrm{Pe}_{E}$ and $\mathrm{Ka}_{E}$ determines whether the value of $\tau_{E}$ is dominated by the convective term, the diffusive term, or the reactive term. The two curves in Figure 2 show the behaviour of $\tau_{E}$ for two possible choices of the problem coefficients. The curves are parametrized by the diameter $h_{E}$ with decreasing values from left to right along each curve. We see that for small values of $h_{E}$ (right-most part of each curve), $\tau$ falls in the diffusive regime, possibly passing through the convective regime, as expected.

\section{Error Analysis}

In the following we assume that the problem is well written in the non-dimensional way, and consequently $\mathrm{K}_{E} \leq 1, h_{E} \leq 1, \beta_{E}=O(1), \gamma_{E} \leq O(1)$. Let $h:=\max _{E \in \mathcal{T}_{h}} h_{E}$ and define the following norms:

$$
\begin{aligned}
\|v\|_{\mathrm{K} \beta} & :=\left(a(v, v)+a_{h}(v, v)\right)^{\frac{1}{2}}, \\
\|v\|_{\mathrm{K} \beta \gamma} & :=\left(\|v\|_{\mathrm{K} \beta}^{2}+\left\|\sqrt{\gamma} \Pi_{k-1}^{0} v\right\|^{2}\right)^{\frac{1}{2}},
\end{aligned}
$$




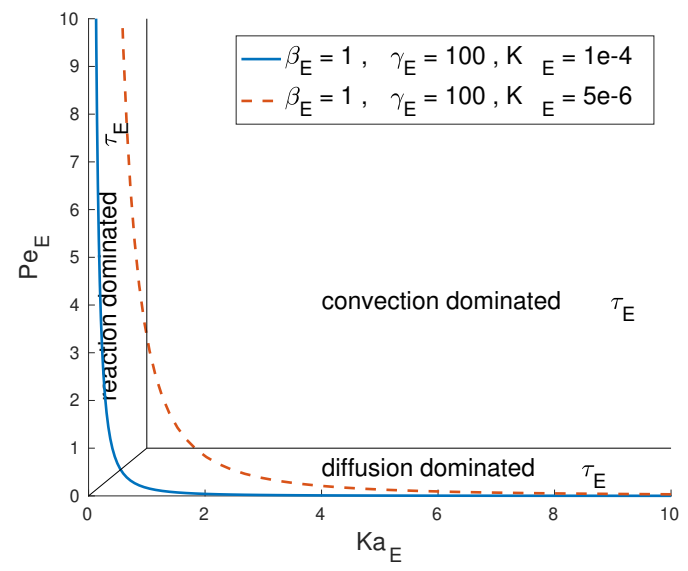

Figure 2: Different regimes of $\tau_{E}$ for different values of $\mathrm{Pe}_{E}$ and $\mathrm{Ka}_{E}$.

on the nonconforming space $\mathrm{H}_{k}^{1, n c}\left(\mathcal{T}_{h}\right)$. In $(36)$, for the evaluation of $S^{E}(v, v)$, we assume to use the VEM interpolant of the function $v$, see [9, Theorem 11]. Clearly, $\|v\|_{\mathrm{K} \beta} \leq$ $\|v\|_{\mathrm{K} \beta \gamma}$.

\subsection{Discretization errors}

The following Lemmas 2, 3, 4, and 5 provide a continuity bound for the discrete bilinear forms (24)-(27) and an estimate of the approximation error when compared with the corresponding continuous ones (14)-(17). Throughout the section, we use the approximation results for the local polynomial projections of a function $v \in \mathrm{H}^{s+1}(E)$, cf. [74, Lemma 5.1], given by:

$$
\begin{array}{rlrl}
\left\|v-\Pi_{k-1}^{0} v\right\|_{E}+h_{E}\left|v-\Pi_{k-1}^{0} v\right|_{1, E} \leq \mathcal{C} h_{E}^{s+1}|v|_{s+1, E} & 1 \leq s+1 \leq k, \\
\left\|v-\Pi_{k}^{\nabla} v\right\|_{E}+h_{E}\left|v-\Pi_{k}^{\nabla} v\right|_{1, E} \leq \mathcal{C} h_{E}^{s+1}|v|_{s+1, E} & 1 \leq s+1 \leq k+1,
\end{array}
$$

which hold for every mesh element $E$ and polynomial degree $k \geq 1$ under the mesh assumptions of Section 3.1. For every internal edge $e=\partial E^{+} \cap \partial E^{-}$and functions $v \in \mathrm{H}^{s+1}\left(\omega_{e}\right)$ with $\omega_{e}=E^{+} \cup E^{-}$we will also consider the trace inequality

$$
\left\|v-\Pi_{k-1}^{0, e}(v)\right\|_{e}+h_{e}\left|v-\Pi_{k-1}^{0, e}(v)\right|_{1, e} \leq \mathcal{C} h_{e}^{s+\frac{1}{2}}|v|_{s+1, \omega_{e}} \quad 1 \leq s+1 \leq k .
$$

We use the error estimate for the virtual element interpolant of order $k$ of a function $\varphi \in \mathrm{H}^{s+1}(E), 1 \leq s+1 \leq k+1[49]$ :

$$
\left\|\varphi-\varphi_{I}\right\|_{E}+h_{E}\left|\varphi-\varphi_{I}\right|_{1, E} \leq \mathcal{C} h_{E}^{s+1}|\varphi|_{s+1, E} .
$$


Furthermore, 41 implies that

$$
\begin{aligned}
\left\|\varphi-\varphi_{I}\right\|_{\mathrm{K} \beta \gamma}^{2}= & \sum_{E \in \mathcal{T}_{h}}\left(\left\|\sqrt{\mathrm{K}} \nabla\left(\varphi-\varphi_{I}\right)\right\|_{E}^{2}+\tau_{E}\left\|\beta \cdot \nabla\left(\varphi-\varphi_{I}\right)\right\|_{E}^{2}+\right. \\
& +\left\|\sqrt{\mathrm{K}} \Pi_{k-1}^{0}\left(\nabla\left(\varphi-\varphi_{I}\right)\right)\right\|_{E}^{2}+\tau_{E}\left\|\beta \cdot \Pi_{k-1}^{0}\left(\nabla\left(\varphi-\varphi_{I}\right)\right)\right\|_{E}^{2} \\
& +S^{E}\left(\left(I-\Pi_{k-1}^{0}\right)\left(\varphi-\varphi_{I}\right),\left(I-\Pi_{k-1}^{0}\right)\left(\varphi-\varphi_{I}\right)\right) \\
& \left.+\left\|\sqrt{\gamma} \Pi_{k-1}^{0}\left(\varphi-\varphi_{I}\right)\right\|_{E}^{2}\right) \\
\leq & \mathcal{C} \sum_{E \in \mathcal{T}_{h}} \max \left\{\mathrm{K}_{E}, \tau_{E} \beta_{E}^{2}, h_{E}^{2} \gamma_{E}\right\} h_{E}^{2 s}|\varphi|_{s+1, E}^{2},
\end{aligned}
$$

for some positive constant $\mathcal{C}$ independent of $h$ and the local problem coefficients $\mathrm{K}, \beta, \gamma$. Assumption 1. We assume that the solution $u$ to $(19)$ belongs to $\mathrm{H}^{s+1}\left(\mathcal{T}_{h}\right) \cap V$, with $1<s+1 \leq k+1$ and that $\mathrm{K} \in\left[\mathrm{W}_{\infty}^{s}(\Omega)\right]^{d \times d}, \beta \in\left[\mathrm{W}_{\infty}^{s+1}(\Omega)\right]^{d}, \gamma \in\left[\mathrm{W}_{\infty}^{s+1}(\Omega)\right]$.

The following technical lemma is needed in the upcoming proofs.

Lemma 1. Let $a, b \in \mathrm{W}_{\infty}^{s}(E)$ be given, $E \in \mathcal{T}_{h}$. Then,

$$
\left\|a b-\Pi_{0}^{0}(a b)\right\|_{\mathrm{W}_{\infty}^{s}(E)} \leq \frac{3}{2}\left(\|a\|_{\mathrm{W}_{\infty}^{s}(E)}\left\|b-\Pi_{0}^{0} b\right\|_{\mathrm{W}_{\infty}^{s}(E)}+\|b\|_{\mathrm{W}_{\infty}^{s}(E)}\left\|a-\Pi_{0}^{0} a\right\|_{\mathrm{W}_{\infty}^{s}(E)}\right) .
$$

Proof. We consider the following decomposition, exploiting the fact that $\Pi_{0}^{0} a \Pi_{0}^{0} b=$ $\Pi_{0}^{0}\left(a \Pi_{0}^{0} b\right)=\Pi_{0}^{0}\left(b \Pi_{0}^{0} a\right)$ :

$$
\begin{aligned}
a b-\Pi_{0}^{0}(a b)= & \frac{1}{2}\left(a b+a b-\Pi_{0}^{0}(a b)-\Pi_{0}^{0}(a b)\right) \\
= & \frac{1}{2}\left[a b-\Pi_{0}^{0}(a) b+\Pi_{0}^{0}(a) b-\Pi_{0}^{0}(a) \Pi_{0}^{0}(b)+\Pi_{0}^{0}\left(b \Pi_{0}^{0}(a)\right)-\Pi_{0}^{0}(a b)\right. \\
& \left.+a b-a \Pi_{0}^{0}(b)+a \Pi_{0}^{0}(b)-\Pi_{0}^{0}(a) \Pi_{0}^{0}(b)+\Pi_{0}^{0}\left(a \Pi_{0}^{0}(b)\right)-\Pi_{0}^{0}(a b)\right] \\
= & \frac{1}{2}\left[\left(a-\Pi_{0}^{0} a\right) b+\left(b-\Pi_{0}^{0} b\right) \Pi_{0}^{0} a+a\left(b-\Pi_{0}^{0} b\right)+\left(a-\Pi_{0}^{0} a\right) \Pi_{0}^{0} b\right. \\
& \left.\Pi_{0}^{0}\left(\left(\Pi_{0}^{0} a-a\right) b\right)+\Pi_{0}^{0}\left(\left(\Pi_{0}^{0} b-b\right) a\right)\right] .
\end{aligned}
$$

The proof is concluded by the triangle inequality, the Cauchy-Schwarz inequality and by exploiting the fact that $\left\|\Pi_{0}^{0} a\right\|_{\mathrm{W}_{\infty}^{s}(E)}=\left|\left(\Pi_{0}^{0} a\right)\right|_{E} \mid \leq\|a\|_{\infty, E} \leq\|a\|_{\mathrm{W}_{\infty}^{s}(E)}$.

We now estimate the terms inside $B_{\mathrm{supg}, h}$ to analyse their continuity, and their consistency with respect to polynomials of order $k$.

Lemma 2. For every function $w \in \mathrm{H}_{k}^{1, n c}\left(\mathcal{T}_{h}\right)$ and $v_{h} \in V_{h} \subset \mathrm{H}_{k}^{1, n c}\left(\mathcal{T}_{h}\right)$,

$$
a_{h}\left(w, v_{h}\right) \leq\|w\|_{\mathrm{K} \beta \gamma}\left\|v_{h}\right\|_{\mathrm{K} \beta \gamma} .
$$

Moreover, if $w \in \mathrm{H}_{k}^{1, n c}\left(\mathcal{T}_{h}\right) \cap \mathrm{H}^{s+1}\left(\mathcal{T}_{h}\right)$, then

$$
\left|a\left(\Pi_{k-1}^{0} w, v_{h}\right)-a_{h}\left(\Pi_{k-1}^{0} w, v_{h}\right)\right| \leq \mathcal{C} \max _{E \in \mathcal{T}_{h}}\left\{\mathcal{C}_{a, E}^{n c}\right\} h^{s}\|w\|_{s+1}\left\|v_{h}\right\|_{\mathrm{K} \beta},
$$


where

$$
\mathcal{C}_{a, E}^{n c}=\frac{\left\|\mathrm{K}_{\beta, E}-\Pi_{0}^{0}\left(\mathrm{~K}_{\beta, E}\right)\right\|_{\mathrm{W}_{\infty}^{s}(E)}}{\sqrt{\mathrm{K}_{E}^{V}}} .
$$

Proof. Regarding (44), to estimate the continuity of $a_{h}$, we use the Cauchy-Schwarz and Hölder inequalities and the definition of the norm (36):

$$
a_{h}\left(w, v_{h}\right) \leq\left(a_{h}(w, w) a_{h}\left(v_{h}, v_{h}\right)\right)^{\frac{1}{2}} \leq\|w\|_{\mathbf{K} \beta}\left\|v_{h}\right\|_{\mathbf{K} \beta} .
$$

To prove 455), we first notice that $S^{E}\left(\left(I-\Pi_{k-1}^{0}\right) \Pi_{k-1}^{0} w, v_{h}\right)=0$ and $\Pi_{k-1}^{0} \nabla \Pi_{k-1}^{0} w=$ $\nabla \Pi_{k-1}^{0} w$ :

$$
\begin{aligned}
& \left|a\left(\Pi_{k-1}^{0} w, v_{h}\right)-a_{h}\left(\Pi_{k-1}^{0} w, v_{h}\right)\right| \leq \sum_{E \in \mathcal{T}_{h}} \mid\left(\mathrm{K}_{\beta, E} \nabla \Pi_{k-1}^{0} w, \nabla v_{h}\right)_{E} \\
& \quad-\left(\mathrm{K}_{\beta, E} \Pi_{k-1}^{0} \nabla \Pi_{k-1}^{0} w, \Pi_{k-1}^{0} \nabla v_{h}\right)_{E}\left|=\sum_{E \in \mathcal{T}_{h}}\right|\left(\mathrm{K}_{\beta, E} \nabla \Pi_{k-1}^{0} w, \nabla v_{h}-\Pi_{k-1}^{0} \nabla v_{h}\right)_{E} \mid .
\end{aligned}
$$

The local terms are bounded using the $k$-consistency $a_{h}^{E}(\cdot, \cdot)=a^{E}(\cdot, \cdot)$ when the coefficients are constants and one of the arguments is a polynomial:

$$
\begin{aligned}
& \left(\mathrm{K}_{\beta, E} \nabla \Pi_{k-1}^{0} w, \nabla v_{h}-\Pi_{k-1}^{0} \nabla v_{h}\right)_{E}=\left(\left(\mathrm{K}_{\beta, E}-\Pi_{0}^{0}\left(\mathrm{~K}_{\beta, E}\right)\right) \nabla \Pi_{k-1}^{0} w, \nabla v_{h}-\Pi_{k-1}^{0} \nabla v_{h}\right)_{E} \\
& =\left(\left(\mathrm{K}_{\beta, E}-\Pi_{0}^{0}\left(\mathrm{~K}_{\beta, E}\right)\right) \nabla \Pi_{k-1}^{0} w-\Pi_{k-1}^{0}\left(\left(\mathrm{~K}_{\beta, E}-\Pi_{0}^{0}\left(\mathrm{~K}_{\beta, E}\right)\right) \nabla \Pi_{k-1}^{0} w\right), \nabla v_{h}-\Pi_{k-1}^{0} \nabla v_{h}\right)_{E} \\
& \leq\left\|\left(\mathrm{K}_{\beta, E}-\Pi_{0}^{0}\left(\mathrm{~K}_{\beta, E}\right)\right) \nabla \Pi_{k-1}^{0} w-\Pi_{k-1}^{0}\left(\left(\mathrm{~K}_{\beta, E}-\Pi_{0}^{0}\left(\mathrm{~K}_{\beta, E}\right)\right) \nabla \Pi_{k-1}^{0} w\right)\right\|_{E}\left\|\nabla v_{h}-\Pi_{k-1}^{0} \nabla v_{h}\right\|_{E} \\
& \leq \mathcal{C} h_{E}^{s}\left|\left(\mathrm{~K}_{\beta, E}-\Pi_{0}^{0}\left(\mathrm{~K}_{\beta, E}\right)\right) \nabla \Pi_{k-1}^{0} w\right|_{s, E}\left(\left\|\nabla v_{h}\right\|_{E}+\left\|\Pi_{k-1}^{0} \nabla v_{h}\right\|_{E}\right) \\
& \leq \mathcal{C} h_{E}^{s} \frac{\left\|\mathrm{K}_{\beta, E}-\Pi_{0}^{0}\left(\mathrm{~K}_{\beta, E}\right)\right\|_{\mathrm{W}_{\infty}^{s}(E)}\|w\|_{s+1, E}\left\|v_{h}\right\|_{\mathrm{K}_{\beta, E}} .}{\sqrt{\mathrm{K}_{E}^{V}}}
\end{aligned}
$$

Remark 5. We can bound $\mathcal{C}_{a, E}^{n c}$ as follows, using (43) and (35):

$$
\begin{aligned}
\mathcal{C}_{a, E}^{n c} & =\frac{\left\|\mathrm{K}_{\beta, E}-\Pi_{0}^{0}\left(\mathrm{~K}_{\beta, E}\right)\right\|_{\mathrm{W}_{\infty}^{s}(E)}}{\sqrt{\mathrm{K}_{E}^{\vee}}} \\
& \leq \frac{1}{\sqrt{\mathrm{K}_{E}^{V}}}\left(\left\|\mathrm{~K}-\Pi_{0}^{0} \mathrm{~K}\right\|_{\mathrm{W}_{\infty}^{s}(E)}+\tau_{E}\left\|\beta \beta^{\top}-\Pi_{0}^{0}\left(\beta \beta^{\top}\right)\right\|_{\mathrm{W}_{\infty}^{s}(E)}\right) \\
& \leq \frac{\mathcal{C}}{\sqrt{\mathrm{K}_{E}^{V}}}\left(\left\|\mathrm{~K}-\Pi_{0}^{0} \mathrm{~K}\right\|_{\mathrm{W}_{\infty}^{s}(E)}+\frac{h_{E}}{\beta_{E}}\|\beta\|_{\mathrm{W}_{\infty}^{s}(E)}\left\|\beta-\Pi_{0}^{0}(\beta)\right\|_{\mathrm{W}_{\infty}^{s}(E)}\right) .
\end{aligned}
$$

Lemma 3. For every function $w \in \mathrm{H}_{k}^{1, n c}\left(\mathcal{T}_{h}\right)$ and $v_{h} \in V_{h} \subset \mathrm{H}_{k}^{1, n c}\left(\mathcal{T}_{h}\right)$ it holds that

$$
\left|b_{h}\left(w, v_{h}\right)\right| \leq \mathcal{C}\left[\max _{E \in \mathcal{T}_{h}}\left(\tau_{E}^{-\frac{1}{2}}, h_{E}^{-1} \mathcal{K}_{b, E}^{n c}\right)\|w\|+\max _{E \in \mathcal{T}_{h}}\left(\mathcal{C}_{b, E}^{n c, 1}, \mathcal{K}_{b, E}^{n c}\right)\|\nabla w\|\right]\left\|v_{h}\right\|_{\mathrm{K} \beta},
$$


where

$$
\begin{aligned}
\mathcal{C}_{b, E}^{n c, r} & =\frac{h_{E}\left\|\beta-\Pi_{0}^{0} \beta\right\|_{\mathrm{W}_{\infty}^{r}(E)}}{\sqrt{\mathrm{K}_{E}^{\vee}}}, r \geq 1, \\
\mathcal{K}_{b, E}^{n c} & =\frac{h_{E}\|\beta \cdot \hat{n}\|_{\mathrm{W}_{\infty}^{1}(\partial E)}}{\sqrt{\mathrm{K}_{E}^{\vee}}} .
\end{aligned}
$$

Moreover, for every function $w \in \mathrm{H}_{k}^{1, n c}\left(\mathcal{T}_{h}\right) \cap \mathrm{H}^{s+1}\left(\mathcal{T}_{h}\right)$, it holds that

$$
\left|b\left(\Pi_{k-1}^{0} w, v_{h}\right)-b_{h}\left(\Pi_{k-1}^{0} w, v_{h}\right)\right| \leq \mathcal{C} \max _{E \in \mathcal{T}_{h}} \mathcal{C}_{b, E}^{n c, s+1} h^{s}\|w\|_{s+1}\left\|v_{h}\right\|_{\boldsymbol{K} \beta} .
$$

Proof. To obtain (47), we first introduce the following decomposition:

$$
b_{h}\left(w, v_{h}\right)=\sum_{E \in \mathcal{T}_{h}} \mathrm{~T}_{E, 1}+\mathrm{T}_{E, 2}+\mathrm{T}_{E, 3}+\mathrm{T}_{E, 4},
$$

where, $\forall E \in \mathcal{T}_{h}$,

$$
\begin{aligned}
& \mathrm{T}_{E, 1}=\left(\beta \cdot\left(\Pi_{k-1}^{0} \nabla w-\nabla w\right), \Pi_{k-1}^{0} v_{h}\right)_{E}, \\
& \mathrm{~T}_{E, 2}=\left(\beta \cdot \nabla w, \Pi_{k-1}^{0} v_{h}-v_{h}\right)_{E}, \\
& \mathrm{~T}_{E, 3}=\left(\beta \cdot \nabla w, v_{h}\right)_{E}, \\
& \mathrm{~T}_{E, 4}=-\left(\Pi_{k-1}^{0} w, \beta \cdot \Pi_{k-1}^{0} \nabla v_{h}\right)_{E} .
\end{aligned}
$$

We estimate $\mathbf{T}_{E, 1}$ in (51) as follows:

$$
\begin{aligned}
\left|\mathrm{T}_{E, 1}\right| & =\left|\left(\Pi_{k-1}^{0} \nabla w-\nabla w,\left(\beta-\Pi_{0}^{0} \beta\right) \Pi_{k-1}^{0} v_{h}\right)_{E}\right| \\
& =\left|\left(\nabla w,\left(\beta-\Pi_{0}^{0} \beta\right) \Pi_{k-1}^{0} v_{h}-\Pi_{k-1}^{0}\left(\left(\beta-\Pi_{0}^{0} \beta\right) \Pi_{k-1}^{0} v_{h}\right)\right)_{E}\right| \\
& \leq\|\nabla w\|_{E}\left\|\left(\beta-\Pi_{0}^{0} \beta\right) \Pi_{k-1}^{0} v_{h}-\Pi_{k-1}^{0}\left(\left(\beta-\Pi_{0}^{0} \beta\right) \Pi_{k-1}^{0} v_{h}\right)\right\|_{E} \\
& \leq \mathcal{C} h_{E}\|\nabla w\|_{E}\left|\left(\beta-\Pi_{0}^{0} \beta\right) \Pi_{k-1}^{0} v_{h}\right|_{1, E} \\
& \leq \mathcal{C} h_{E}\|\nabla w\|_{E}\left\|\beta-\Pi_{0}^{0} \beta\right\|_{\mathrm{W}_{\infty}^{1}(E)}\left\|\Pi_{k-1}^{0} v_{h}\right\|_{1, E} \\
& \leq \mathcal{C} \frac{h_{E}\left\|\beta-\Pi_{0}^{0} \beta\right\|_{\mathrm{W}_{\infty}^{1}(E)}\|\nabla w\|_{E}\left\|v_{h}\right\|_{\mathrm{K} \beta} .}{\sqrt{\mathrm{K}_{E}^{V}}}
\end{aligned}
$$


$\mathrm{T}_{E, 2}$ in 52 is estimated similarly, using also 29):

$$
\begin{aligned}
\left|\mathrm{T}_{E, 2}\right|= & \left|\left(\beta \cdot \nabla w, \Pi_{k-1}^{0} v_{h}-v_{h}\right)_{E}\right| \\
\leq & \left|\left(w, \beta \cdot \nabla\left(\Pi_{k-1}^{0} v_{h}-v_{h}\right)\right)_{E}\right|+\left|\int_{\partial E} w(\beta \cdot \hat{n})\left(\Pi_{k-1}^{0} v_{h}-v_{h}\right)\right| \\
\leq & \tau_{E}^{-\frac{1}{2}}\|w\|_{E} \tau_{E}^{\frac{1}{2}}\left\|\beta \cdot \nabla\left(\Pi_{k-1}^{0} v_{h}-v_{h}\right)\right\|_{E} \\
& +\mathcal{C}\|w\|_{\partial E}\|\beta \cdot \hat{n}\|_{\infty, \partial E}\left\|v_{h}-\Pi_{k-1}^{0} v_{h}\right\|_{\partial E} \\
\leq & \tau_{E}^{-\frac{1}{2}}\|w\|_{E} \cdot \sqrt{\frac{1}{\sigma_{*}} \tau_{E} \beta_{E}^{2} S_{*}^{E}\left(\left(I-\Pi_{k-1}^{0}\right) v_{h},\left(I-\Pi_{k-1}^{0}\right) v_{h}\right)} \\
& +\mathcal{C} h_{E}^{-\frac{1}{2}}\|w\|_{E}\|\beta \cdot \hat{n}\|_{\infty, \partial E} \cdot h_{E}^{\frac{1}{2}}\left\|\nabla v_{h}\right\|_{E} \\
\leq & \mathcal{C}\left(\tau_{E}^{-\frac{1}{2}}+\frac{\|\beta \cdot \hat{n}\|_{\infty, \partial E}}{\sqrt{\mathrm{K}_{E}^{\vee}}}\right)\|w\|_{E}\left\|v_{h}\right\|_{\mathrm{K} \beta, E} .
\end{aligned}
$$

The estimation of $\mathrm{T}_{E, 3}$ in 53 requires an application of Green's formula, as follows:

$$
\begin{aligned}
\left|\sum_{E \in \mathcal{T}_{h}} \mathrm{~T}_{E, 3}\right| & \leq\left|\sum_{E \in \mathcal{T}_{h}}\left(w, \beta \cdot \nabla v_{h}\right)_{E}\right|+\left|\sum_{E \in \mathcal{T}_{h}} \int_{\partial E}(\beta \cdot \hat{n}) w v_{h}\right| \\
& =\left|\sum_{E \in \mathcal{T}_{h}}\left(w, \beta \cdot \nabla v_{h}\right)_{E}\right|+\left|\frac{1}{2} \sum_{E \in \mathcal{T}_{h}} \int_{\partial E}(\beta \cdot \hat{n}) \llbracket w v_{h} \rrbracket\right| .
\end{aligned}
$$

The first term is estimated locally by the Cauchy-Schwarz inequality:

$$
\left(w, \beta \cdot \nabla v_{h}\right)_{E} \leq \tau_{E}^{-\frac{1}{2}}\|w\|_{E}\left\|v_{h}\right\|_{\mathrm{K} \beta, E} .
$$

The boundary terms are estimated exploiting $\int_{\partial E} \beta \cdot \hat{n}=\int_{E} \nabla \cdot \beta=0$, and denoting by 
$\Pi_{k-1}^{0, \partial E} v$ the piecewise polynomial projection of $v$ on each $e \subset \partial E$ :

$$
\begin{aligned}
& \int_{\partial E}(\beta \cdot \hat{n}) \llbracket w v_{h} \rrbracket=\sum_{R \in \omega_{E}} \int_{E \cap R}(\beta \cdot \hat{n})\left(\left.w\right|_{R} \llbracket v_{h} \rrbracket+\left.\llbracket w \rrbracket v_{h}\right|_{E}\right) \\
& =\sum_{R \in \omega_{E}} \int_{E \cap R}\left(\left.(\beta \cdot \hat{n}) w\right|_{R}-\Pi_{k-1}^{0, \partial E}\left(\left.(\beta \cdot \hat{n}) w\right|_{R}\right)\right) \llbracket v_{h} \rrbracket \\
& +\sum_{R \in \omega_{E}} \int_{E \cap R} \llbracket w \rrbracket\left(\left.(\beta \cdot \hat{n}) v_{h}\right|_{E}-\Pi_{k-1}^{0, \partial E}\left(\left.(\beta \cdot \hat{n}) v_{h}\right|_{E}\right)\right) \\
& =\sum_{R \in \omega_{E}} \int_{E \cap R}\left(\left.(\beta \cdot \hat{n}) w\right|_{R}-\Pi_{k-1}^{0, \partial E}\left(\left.(\beta \cdot \hat{n}) w\right|_{R}\right)\right) \llbracket v_{h}-\Pi_{k-1}^{0, \partial E}\left(v_{h}\right) \rrbracket \\
& +\sum_{R \in \omega_{E}} \int_{E \cap R} \llbracket w-\Pi_{k-1}^{0} w \rrbracket\left(\left.(\beta \cdot \hat{n}) v_{h}\right|_{E}-\Pi_{k-1}^{0, \partial E}\left(\left.(\beta \cdot \hat{n}) v_{h}\right|_{E}\right)\right) \\
& =\sum_{R \in \omega_{E}}\left\|\left.(\beta \cdot \hat{n}) w\right|_{R}-\Pi_{k-1}^{0, \partial E}\left(\left.(\beta \cdot \hat{n}) w\right|_{R}\right)\right\|_{R \cap E}\left\|\llbracket v_{h}-\Pi_{k-1}^{0, \partial E}\left(v_{h}\right) \rrbracket\right\|_{E \cap R} \\
& +\sum_{R \in \omega_{E}}\left\|\llbracket w-\Pi_{k-1}^{0} w \rrbracket\right\|_{E \cap R}\left\|\left.(\beta \cdot \hat{n}) v_{h}\right|_{E}-\Pi_{k-1}^{0, \partial E}\left(\left.(\beta \cdot \hat{n}) v_{h}\right|_{E}\right)\right\|_{E \cap R} \\
& \leq \mathcal{C}_{1}\left(\left.\sum_{R \in \omega_{E}} h_{E}|(\beta \cdot \hat{n}) w|_{R}\right|_{1, R \cap E}\right) h_{E}^{\frac{1}{2}}\left\|\nabla v_{h}\right\|_{\omega_{E}} \\
& +\mathcal{C}_{2}\left(\left.\sum_{R \in \omega_{E}} h_{E}\left|(\beta \cdot \hat{n}) v_{h}\right|_{E}\right|_{1, E \cap R}\right) h_{E}^{\frac{1}{2}}\|\nabla w\|_{\omega_{E}} \\
& \leq \mathcal{C}_{1} h_{E}^{\frac{1}{2}}\|\beta \cdot \hat{n}\|_{\mathrm{W}_{\infty}^{1}(\partial E)}\|\nabla w\|_{\omega_{E}} \cdot h_{E}^{\frac{1}{2}}\left\|\nabla v_{h}\right\|_{\omega_{E}} \\
& +\mathcal{C}_{2} h_{E}^{\frac{1}{2}}\|\beta \cdot \hat{n}\|_{\mathrm{W}_{\infty}^{1}(\partial E)}\left\|\nabla v_{h}\right\|_{\omega_{E}} \cdot h_{E}^{\frac{1}{2}}\|\nabla w\|_{\omega_{E}} \\
& \leq \mathcal{C} \frac{h_{E}\|\beta \cdot \hat{n}\|_{\mathrm{W}_{\infty}^{1}(\partial E)}}{\sqrt{\mathrm{K}_{E}^{V}}}\|\nabla w\|_{\omega_{E}}\left\|v_{h}\right\|_{\mathrm{K} \beta, \omega_{E}} .
\end{aligned}
$$

The estimate of $\mathrm{T}_{E, 4}$ defined by (54) is obtained by the Cauchy-Schwarz inequality, the continuity of projections and the definition of the norm (36):

$$
\left|\mathbf{T}_{E, 4}\right|=\left|\left(\Pi_{k-1}^{0} w, \beta \cdot \Pi_{k-1}^{0} \nabla v_{h}\right)_{E}\right| \leq \mathcal{C} \tau_{E}^{-\frac{1}{2}}\|w\|_{E}\left\|v_{h}\right\|_{\mathbf{K} \beta, E} .
$$

To derive (50), we set

$$
b\left(\Pi_{k-1}^{0} w, v_{h}\right)-b_{h}\left(\Pi_{k-1}^{0} w, v_{h}\right)=\sum_{E \in \mathcal{T}_{h}}\left(\mathrm{R}_{E, 1}-\mathrm{R}_{E, 2}\right),
$$

where, recalling that $\Pi_{k-1}^{0}\left(\nabla \Pi_{k-1}^{0} w\right)=\nabla \Pi_{k-1}^{0} w$,

$$
\begin{aligned}
& \mathrm{R}_{E, 1}=\left(\beta \cdot \nabla \Pi_{k-1}^{0} w, v_{h}-\Pi_{k-1}^{0} v_{h}\right)_{E}, \\
& \mathbf{R}_{E, 2}=\left(\Pi_{k-1}^{0} w, \beta \cdot\left(\nabla v_{h}-\Pi_{k-1}^{0} \nabla v_{h}\right)\right)_{E} .
\end{aligned}
$$


$\mathrm{R}_{E, 1}$ can be estimated as follows:

$$
\begin{aligned}
\mathrm{R}_{E, 1} & =\left(\beta \cdot \nabla \Pi_{k-1}^{0} w, v_{h}-\Pi_{k-1}^{0} v_{h}\right)_{E}=\left(\left(\beta-\Pi_{0}^{0} \beta\right) \cdot \nabla \Pi_{k-1}^{0} w, v_{h}-\Pi_{k-1}^{0} v_{h}\right)_{E} \\
& =\left(\left(\beta-\Pi_{0}^{0} \beta\right) \cdot \nabla \Pi_{k-1}^{0} w-\Pi_{k-1}^{0}\left(\left(\beta-\Pi_{0}^{0} \beta\right) \cdot \nabla \Pi_{k-1}^{0} w\right), v_{h}-\Pi_{k-1}^{0} v_{h}\right)_{E} \\
& \leq\left\|\left(\beta-\Pi_{0}^{0} \beta\right) \cdot \nabla \Pi_{k-1}^{0} w-\Pi_{k-1}^{0}\left(\left(\beta-\Pi_{0}^{0} \beta\right) \cdot \nabla \Pi_{k-1}^{0} w\right)\right\|_{E}\left\|v_{h}-\Pi_{k-1}^{0} v_{h}\right\|_{E} \\
& \leq \mathcal{C} h_{E}^{s}\left|\left(\beta-\Pi_{0}^{0} \beta\right) \cdot \nabla \Pi_{k-1}^{0} w\right|_{s, E} \cdot h_{E}\left\|\nabla v_{h}\right\|_{E} \\
& \leq \mathcal{C} h_{E}^{s+1}\left\|\beta-\Pi_{0}^{0} \beta\right\|_{\mathrm{W}_{\infty}^{s}(E)}\|w\|_{s+1, E}\left\|\nabla v_{h}\right\|_{E} \\
& \leq \mathcal{C} \frac{h_{E}\left\|\beta-\Pi_{0}^{0} \beta\right\|_{\mathrm{W}_{\infty}^{s}(E)}}{\sqrt{\mathrm{K}_{E}^{V}}} h_{E}^{s}\|w\|_{s+1, E}\left\|v_{h}\right\|_{\mathrm{K} \beta} .
\end{aligned}
$$

The estimate of $\mathbf{R}_{E, 2}$ in 56 is obtained as follows:

$$
\begin{aligned}
\mathrm{R}_{E, 2} & =\left(\Pi_{k-1}^{0} w, \beta \cdot\left(\nabla v_{h}-\Pi_{k-1}^{0} \nabla v_{h}\right)\right)_{E}=\left(\left(\beta-\Pi_{0}^{0} \beta\right) \Pi_{k-1}^{0} w, \nabla v_{h}-\Pi_{k-1}^{0} \nabla v_{h}\right)_{E} \\
& =\left(\left(\beta-\Pi_{0}^{0} \beta\right) \Pi_{k-1}^{0} w-\Pi_{k-1}^{0}\left(\left(\beta-\Pi_{0}^{0} \beta\right) \Pi_{k-1}^{0} w\right), \nabla v_{h}\right)_{E} \\
& \leq\left\|\left(\beta-\Pi_{0}^{0} \beta\right) \Pi_{k-1}^{0} w-\Pi_{k-1}^{0}\left(\left(\beta-\Pi_{0}^{0} \beta\right) \Pi_{k-1}^{0} w\right)\right\|_{E}\left\|\nabla v_{h}\right\|_{E} \\
& \leq \mathcal{C} h_{E}^{s+1}\left|\left(\beta-\Pi_{0}^{0} \beta\right) \Pi_{k-1}^{0} w\right|_{s+1, E}\left\|\nabla v_{h}\right\|_{E} \\
& \leq \mathcal{C} h_{E}^{s+1}\left\|\beta-\Pi_{0}^{0} \beta\right\|_{\mathrm{W}_{\infty}^{s+1}(E)}\left\|\Pi_{k-1}^{0} w\right\|_{s+1, E}\left\|\nabla v_{h}\right\|_{E} \\
& \leq \mathcal{C} \frac{h_{E}\left\|\beta-\Pi_{0}^{0} \beta\right\|_{\mathrm{W}_{\infty}^{s+1}(E)}}{\sqrt{\mathrm{K}_{E}^{V}}} h_{E}^{s}\|w\|_{s+1, E}\left\|v_{h}\right\|_{\mathrm{K} \beta, E} .
\end{aligned}
$$

Remark 6. The coefficient $\mathcal{K}_{b, E}^{n c}$ can be rewritten, considering that $\overline{\beta \cdot \hat{n}}^{\partial E}=\int_{\partial E} \beta \cdot \hat{n}=0$, in the following way:

$$
\mathcal{K}_{b, E}^{n c}=\frac{h_{E}\|\beta \cdot \hat{n}\|_{\mathrm{W}_{\infty}^{1}(\partial E)}}{\sqrt{\mathrm{K}_{E}^{V}}}=\frac{h_{E}\|\beta \cdot \hat{n}-\overline{\beta \cdot \hat{n}} \partial E\|_{\mathrm{W}_{\infty}^{1}(\partial E)}}{\sqrt{\mathrm{K}_{E}^{V}}}
$$

Lemma 4. For every function $w \in \mathrm{H}_{k}^{1, n c}\left(\mathcal{T}_{h}\right)$ and $v_{h} \in V_{h}$ it holds that

$$
\left|c_{h}\left(w, v_{h}\right)\right| \leq\left(1+\sqrt{C_{\tau}}\right)\|w\|_{\mathrm{K} \beta \gamma}\left\|v_{h}\right\|_{\mathrm{K} \beta \gamma} .
$$

Moreover, for every function $w \in \mathrm{H}_{k}^{1, n c}\left(\mathcal{T}_{h}\right) \cap \mathrm{H}^{s+1}\left(\mathcal{T}_{h}\right)$, it holds that

$$
\begin{gathered}
\left|c\left(\Pi_{k-1}^{0} w, v_{h}\right)-c_{h}\left(\Pi_{k-1}^{0} w, v_{h}\right)\right| \leq \mathcal{C} \max _{E \in \mathcal{T}_{h}} \mathcal{C}_{c, E}^{n c} h^{s}\|w\|_{s+1}\left\|v_{h}\right\|_{\mathrm{K} \beta}, \\
\mathcal{C}_{c, E}^{n c}=\max \left\{\frac{h_{E}^{2}\left\|\gamma-\Pi_{0}^{0} \gamma\right\|_{\mathrm{W}_{\infty}^{s+1}(E)}}{\sqrt{\mathrm{K}_{E}^{V}}}, \frac{h_{E} \tau_{E}\left\|\gamma \beta-\Pi_{0}^{0}(\gamma \beta)\right\|_{\mathrm{W}_{\infty}^{s+1}(E)}}{\sqrt{\mathrm{K}_{E}^{V}}}\right\}
\end{gathered}
$$


Proof. Inequality (57) follows easily from the definition of the norm (37) and the definition of $\tau_{E}$ (35):

$$
\begin{aligned}
& \left(\gamma \Pi_{k-1}^{0} w, \Pi_{k-1}^{0} v_{h}\right)_{E}+\tau_{E}\left(\gamma \Pi_{k-1}^{0} w, \beta \cdot \Pi_{k-1}^{0} \nabla v_{h}\right)_{E} \leq\left\|\sqrt{\gamma} \Pi_{k-1}^{0} w\right\|_{E}\left\|\sqrt{\gamma} \Pi_{k-1}^{0} v_{h}\right\|_{E} \\
& \quad+\sqrt{\tau_{E} \gamma_{E}}\left\|\sqrt{\gamma} \Pi_{k-1}^{0} w\right\|_{E} \cdot \sqrt{\tau_{E}}\left\|\beta \cdot \Pi_{k-1}^{0} \nabla v_{h}\right\|_{E} \leq\left(1+\sqrt{C_{\tau}}\right)\|w\|_{\mathrm{K} \beta \gamma, E}\left\|v_{h}\right\|_{\mathrm{K} \beta \gamma, E} .
\end{aligned}
$$

To prove 58 , we start with:

$$
c\left(\Pi_{k-1}^{0} w, v_{h}\right)-c_{h}\left(\Pi_{k-1}^{0} w, v_{h}\right)=\sum_{E \in \mathcal{T}_{h}}\left(\mathrm{R}_{E, 1}+\mathrm{R}_{E, 2}\right),
$$

where

$$
\begin{aligned}
& \mathrm{R}_{E, 1}=\left(\gamma \Pi_{k-1}^{0} w, v_{h}-\Pi_{k-1}^{0} v_{h}\right)_{E}, \\
& \mathrm{R}_{E, 2}=\tau_{E}\left(\gamma \Pi_{k-1}^{0} w, \beta \cdot \nabla v_{h}-\beta \cdot \Pi_{k-1}^{0} \nabla v_{h}\right)_{E} .
\end{aligned}
$$

The first term, given by 60 , can be bounded as follows:

$$
\begin{aligned}
\mathrm{R}_{E, 1} & =\left(\left(\gamma-\Pi_{0}^{0} \gamma\right) \Pi_{k-1}^{0} w, v_{h}-\Pi_{k-1}^{0} v_{h}\right)_{E} \\
& =\left(\left(\gamma-\Pi_{0}^{0} \gamma\right) \Pi_{k-1}^{0} w-\Pi_{k-1}^{0}\left(\left(\gamma-\Pi_{0}^{0} \gamma\right) \Pi_{k-1}^{0} w\right), v_{h}-\Pi_{k-1}^{0} v_{h}\right)_{E} \\
& \leq\left\|\left(\gamma-\Pi_{0}^{0} \gamma\right) \Pi_{k-1}^{0} w-\Pi_{k-1}^{0}\left(\left(\gamma-\Pi_{0}^{0} \gamma\right) \Pi_{k-1}^{0} w\right)\right\|_{E}\left\|v_{h}-\Pi_{k-1}^{0} v_{h}\right\|_{E} \\
& \leq \mathcal{C} h_{E}^{s+1}\left|\left(\gamma-\Pi_{0}^{0} \gamma\right) \Pi_{k-1}^{0} w\right|_{s+1, E} \cdot h_{E}\left\|\nabla v_{h}\right\|_{E} \\
& \leq \mathcal{C} h_{E}^{s+2}\left\|\gamma-\Pi_{0}^{0} \gamma\right\|_{\mathrm{W}_{\infty}^{s+1}(E)}\left\|\Pi_{k-1}^{0} w\right\|_{s+1, E}\left\|\nabla v_{h}\right\|_{E} \\
& \leq \mathcal{C} \frac{h_{E}^{2}\left\|\gamma-\Pi_{0}^{0} \gamma\right\|_{\mathrm{W}_{\infty}^{s+1}(E)}}{\sqrt{\mathrm{K}_{E}^{V}}} h_{E}^{s}\|w\|_{s+1, E}\left\|v_{h}\right\|_{\mathrm{K} \beta, E} .
\end{aligned}
$$

The term $\mathrm{R}_{E, 2}$ in (56) can be bounded as follows:

$$
\begin{aligned}
\mathrm{R}_{E, 2} & =\tau_{E}\left(\gamma \Pi_{k-1}^{0} w, \beta \cdot \nabla v_{h}-\beta \cdot \Pi_{k-1}^{0} \nabla v_{h}\right)_{E} \\
& =\tau_{E}\left(\left(\gamma \beta-\Pi_{0}^{0}(\gamma \beta)\right) \Pi_{k-1}^{0} w, \nabla v_{h}-\Pi_{k-1}^{0} \nabla v_{h}\right)_{E} \\
& =\tau_{E}\left(\left(\gamma \beta-\Pi_{0}^{0}(\gamma \beta)\right) \Pi_{k-1}^{0} w-\Pi_{k-1}^{0}\left(\left(\gamma \beta-\Pi_{0}^{0}(\gamma \beta)\right) \Pi_{k-1}^{0} w\right), \nabla v_{h}-\Pi_{k-1}^{0} \nabla v_{h}\right)_{E} \\
& \leq \tau_{E}\left\|\left(\gamma \beta-\Pi_{0}^{0}(\gamma \beta)\right) \Pi_{k-1}^{0} w-\Pi_{k-1}^{0}\left(\left(\gamma \beta-\Pi_{0}^{0}(\gamma \beta)\right) \Pi_{k-1}^{0} w\right)\right\|_{E}\left\|\nabla v_{h}-\Pi_{k-1}^{0} \nabla v_{h}\right\|_{E} \\
& \leq \mathcal{C} h_{E}^{s+1} \tau_{E}\left|\left(\gamma \beta-\Pi_{0}^{0}(\gamma \beta)\right) \Pi_{k-1}^{0} w\right|_{s+1, E}\left\|\nabla v_{h}\right\|_{E} \\
& \leq \mathcal{C} h_{E} \tau_{E}\left\|\gamma \beta-\Pi_{0}^{0}(\gamma \beta)\right\|_{\mathrm{W}_{\infty}^{s+1}(E)} h_{E}^{s}\|w\|_{s+1, E}\left\|\nabla v_{h}\right\|_{E} \\
& \leq \mathcal{C} \frac{h_{E} \tau_{E}\left\|\gamma \beta-\Pi_{0}^{0}(\gamma \beta)\right\|_{\mathrm{W}_{\infty}^{s+1}(E)}}{\sqrt{\mathrm{K}_{E}^{V}}} h_{E}^{s}\|w\|_{s+1, E}\left\|v_{h}\right\|_{\mathrm{K} \beta, E} .
\end{aligned}
$$

Remark 7. The second argument of the max in $(59)$ can be bounded by 43 and 35 :

$$
\begin{array}{r}
\frac{h_{E} \tau_{E}\left\|\gamma \beta-\Pi_{0}^{0}(\gamma \beta)\right\|_{\mathrm{W}_{\infty}^{s+1}(E)} \leq \frac{\mathcal{C}}{\sqrt{\mathrm{K}_{E}^{V}}}\left(\frac{C_{\tau} h_{E}}{\gamma_{E}}\|\gamma\|_{\mathrm{W}_{\infty}^{s+1}(E)}\left\|\beta-\Pi_{0}^{0} \beta\right\|_{\mathrm{W}_{\infty}^{s+1}(E)}\right.}{19} \\
\left.+\frac{h_{E}^{2}}{\beta_{E}}\|\beta\|_{\mathrm{W}_{\infty}^{s+1}(E)}\left\|\gamma-\Pi_{0}^{0} \gamma\right\|_{\mathrm{W}_{\infty}^{s+1}(E)}\right)
\end{array}
$$


Lemma 5. For any $w \in \mathrm{H}_{k}^{1, n c}\left(\mathcal{T}_{h}\right)$ and $\forall v_{h} \in V_{h}$,

$$
d_{h}\left(w, v_{h}\right) \leq\|w\|_{\mathrm{K} \beta}\left\|v_{h}\right\|_{\mathrm{K} \beta} .
$$

Moreover, if $w \in V \cap \mathrm{H}^{s+1}\left(\mathcal{T}_{h}\right)$, then

$$
\left|d\left(\Pi_{k-1}^{0} w, v_{h}\right)-d_{h}\left(\Pi_{k-1}^{0} w, v_{h}\right)\right| \leq \mathcal{C} \max _{E \in \mathcal{T}_{h}} \mathcal{C}_{d, E}^{n c} h_{E}^{s}\|w\|_{s+1}\left\|v_{h}\right\|_{\mathrm{K} \beta},
$$

where

$$
\mathcal{C}_{d, E}^{n c}=\max \left\{\frac{h_{E}^{-1} \tau_{E} \sum_{i=1}^{d}\left\|\beta_{i} \mathrm{~K}-\Pi_{0}^{0}\left(\beta_{i} \mathrm{~K}\right)\right\|_{\mathrm{W}_{\infty}^{s}(E)}}{\sqrt{\mathrm{K}_{E}^{V}}}, \frac{\tau_{E}\left\|(\nabla \beta)^{\top} \mathrm{K}-\Pi_{0}^{0}\left((\nabla \beta)^{\top} \mathrm{K}\right)\right\|_{\mathrm{W}_{\infty}^{s}(E)}}{\sqrt{\mathrm{K}_{E}^{\mathrm{V}}}}\right\} .
$$

Proof. To prove (62), we use the inverse inequality (34) and the definition of the norm (36): $\forall E \in \mathcal{T}_{h}$,

$$
\begin{aligned}
\tau_{E}\left(\nabla \cdot\left(\mathrm{K} \Pi_{k-1}^{0} \nabla w\right), \beta \cdot \Pi_{k-1}^{0} \nabla v_{h}\right)_{E} & \leq \sqrt{\tau_{E}}\left\|\nabla \cdot\left(\mathrm{K} \Pi_{k-1}^{0} \nabla w\right)\right\|_{E} \cdot \sqrt{\tau_{E}}\left\|\beta \cdot \Pi_{k-1}^{0} \nabla v_{h}\right\|_{E} \\
& \leq \frac{1}{\sqrt{\mathrm{K}_{E}}}\left\|\mathrm{~K} \Pi_{k-1}^{0} \nabla w\right\|_{E}\left\|v_{h}\right\|_{\mathrm{K} \beta} \leq\|w\|_{\mathrm{K} \beta}\left\|v_{h}\right\|_{\mathrm{K} \beta} .
\end{aligned}
$$

Regarding 63), we procede as follows: $\forall E \in \mathcal{T}_{h}$,

$$
\tau_{E}\left(\nabla \cdot\left(\mathrm{K} \Pi_{k-1}^{0} \nabla w\right), \beta \cdot\left(\nabla v_{h}-\Pi_{k-1}^{0} \nabla v_{h}\right)\right)_{E}=\mathrm{R}_{E, 1}+\mathrm{R}_{E, 2},
$$

where, with the notation $\mathcal{E}_{k-1}=I-\Pi_{k-1}^{0}$,

$$
\begin{aligned}
& \mathrm{R}_{E, 1}=\tau_{E} \sum_{i=1}^{d}\left(\nabla \cdot\left(\beta_{i} \mathrm{~K} \Pi_{k-1}^{0} \nabla w\right), \mathcal{E}_{k-1}\left(\frac{\partial v_{h}}{\partial x_{i}}\right)\right)_{E}, \\
& \mathrm{R}_{E, 2}=\tau_{E}\left(-(\nabla \beta)^{\top} \mathrm{K} \Pi_{k-1}^{0} \nabla w, \mathcal{E}_{k-1}\left(\nabla v_{h}\right)\right)_{E} .
\end{aligned}
$$


The term $\mathrm{R}_{E, 1}$ in 55 can be estimated as follows, using (43),

$$
\begin{aligned}
\mathrm{R}_{E, 1} & =\tau_{E} \sum_{i=1}^{d}\left(\nabla \cdot\left(\left(\beta_{i} \mathrm{~K}-\Pi_{0}^{0}\left(\beta_{i} \mathrm{~K}\right)\right) \Pi_{k-1}^{0} \nabla w\right), \mathcal{E}_{k-1}\left(\frac{\partial v_{h}}{\partial x_{i}}\right)\right)_{E} \\
& =\tau_{E} \sum_{i=1}^{d}\left(\nabla \cdot\left(\mathcal{E}_{k-1}\left(\left(\beta_{i} \mathrm{~K}-\Pi_{0}^{0}\left(\beta_{i} \mathrm{~K}\right)\right) \Pi_{k-1}^{0} \nabla w\right)\right), \mathcal{E}_{k-1}\left(\frac{\partial v_{h}}{\partial x_{i}}\right)\right)_{E} \\
& \leq \tau_{E} \sum_{i=1}^{d}\left\|\nabla \cdot\left(\mathcal{E}_{k-1}\left(\left(\beta_{i} \mathrm{~K}-\Pi_{0}^{0}\left(\beta_{i} \mathrm{~K}\right)\right) \Pi_{k-1}^{0} \nabla w\right)\right)\right\|_{E}\left\|\mathcal{E}_{k-1}\left(\frac{\partial v_{h}}{\partial x_{i}}\right)\right\|_{E} \\
& \leq \mathcal{C} h_{E}^{-1} \tau_{E} \sum_{i=1}^{d}\left\|\mathcal{E}_{k-1}\left(\left(\beta_{i} \mathrm{~K}-\Pi_{0}^{0}\left(\beta_{i} \mathrm{~K}\right)\right) \Pi_{k-1}^{0} \nabla w\right)\right\|_{E}\left\|\frac{\partial v_{h}}{\partial x_{i}}\right\|_{E} \\
& \leq \mathcal{C} h_{E}^{-1} \tau_{E} h_{E}^{s} \sum_{i=1}^{d}\left|\left(\beta_{i} \mathrm{~K}-\Pi_{0}^{0}\left(\beta_{i} \mathrm{~K}\right)\right) \Pi_{k-1}^{0} \nabla w\right|_{s, E}\left\|\nabla v_{h}\right\|_{E} \\
& \leq \mathcal{C} h_{E}^{-1} \tau_{E} h_{E}^{s}\left(\sum_{i=1}^{d}\left\|\beta_{i} \mathrm{~K}-\Pi_{0}^{0}\left(\beta_{i} \mathrm{~K}\right)\right\|_{\mathrm{W}_{\infty}^{s}(E)}\right)\left\|\Pi_{k-1}^{0} \nabla w\right\|_{s, E}\left\|\nabla v_{h}\right\|_{E} \\
& \leq \mathcal{C} \frac{h_{E}^{-1} \tau_{E} \sum_{i=1}^{d}\left\|\beta_{i} \mathrm{~K}-\Pi_{0}^{0}\left(\beta_{i} \mathrm{~K}\right)\right\|_{\mathrm{W}_{\infty}^{s}(E)} h_{E}^{s}\|w\|_{s+1, E}\left\|v_{h}\right\|_{\mathrm{K} \beta, E} .}{\sqrt{\mathrm{K}_{E}^{\vee}}}
\end{aligned}
$$

The term $\mathrm{R}_{E, 2}$ in (56) can be estimated as follows, using also (43):

$$
\begin{aligned}
\left|\mathrm{R}_{E, 2}\right| & =\tau_{E}\left|\left((\nabla \beta)^{\top} \mathrm{K} \Pi_{k-1}^{0} \nabla w, \mathcal{E}_{k-1}\left(\nabla v_{h}\right)\right)_{E}\right| \\
& =\tau_{E}\left|\left(\mathcal{E}_{k-1}\left(\left((\nabla \beta)^{\top} \mathrm{K}-\Pi_{0}^{0}\left((\nabla \beta)^{\top} \mathrm{K}\right)\right) \Pi_{k-1}^{0} \nabla w\right), \nabla v_{h}\right)_{E}\right| \\
& \leq \tau_{E}\left\|\mathcal{E}_{k-1}\left(\left((\nabla \beta)^{\top} \mathrm{K}-\Pi_{0}^{0}\left((\nabla \beta)^{\top} \mathrm{K}\right)\right) \Pi_{k-1}^{0} \nabla w\right)\right\|_{E}\left\|\nabla v_{h}\right\|_{E} \\
& \leq \mathcal{C} \tau_{E} h_{E}^{s}\left|\left((\nabla \beta)^{\top} \mathrm{K}-\Pi_{0}^{0}\left((\nabla \beta)^{\top} \mathrm{K}\right)\right) \Pi_{k-1}^{0} \nabla w\right|_{s, E}\left\|\nabla v_{h}\right\|_{E} \\
& \leq \mathcal{C} \frac{\tau_{E}\left\|(\nabla \beta)^{\top} \mathrm{K}-\Pi_{0}^{0}\left((\nabla \beta)^{\top} \mathrm{K}\right)\right\|_{\mathrm{W}_{\infty}^{s}(E)}}{\sqrt{\mathrm{K}_{E}^{V}}} h_{E}^{s}\|w\|_{s+1, E}\left\|v_{h}\right\|_{\mathrm{K} \beta, E} .
\end{aligned}
$$

Remark 8. The first argument of the max in 64 can be bounded as follows, using 43 and 35 :

$$
\begin{aligned}
& \frac{h_{E}^{-1} \tau_{E} \sum_{i=1}^{d}\left\|\beta_{i} \mathrm{~K}-\Pi_{0}^{0}\left(\beta_{i} \mathrm{~K}\right)\right\|_{\mathrm{W}_{\infty}^{s}(E)}}{\sqrt{\mathrm{K}_{E}^{V}}} \leq \frac{3 h_{E}^{-1} \tau_{E}}{2 \sqrt{\mathrm{K}_{E}^{V}}}\left(2\|\mathrm{~K}\|_{\mathrm{W}_{\infty}^{s}(E)}\left\|\beta-\Pi_{0}^{0} \beta\right\|_{\mathrm{W}_{\infty}^{s}(E)}\right. \\
& \left.\quad+2\|\beta\|_{\mathrm{W}_{\infty}^{s}(E)}\left\|\mathrm{K}-\Pi_{0}^{0} \mathrm{~K}\right\|_{\mathrm{W}_{\infty}^{s}(E)}\right) \\
& \leq \frac{\mathcal{C}}{\sqrt{\mathrm{K}_{E}^{V}}}\left(h_{E}\left\|\frac{\mathrm{K}}{\mathrm{K}_{E}}\right\|_{\mathrm{W}_{\infty}^{s}(E)}\left\|\beta-\Pi_{0}^{0} \beta\right\|_{\mathrm{W}_{\infty}^{s}(E)}+\left\|\frac{\beta}{\beta_{E}}\right\|_{\mathrm{W}_{\infty}^{s}(E)}\left\|\mathrm{K}-\Pi_{0}^{0} \mathrm{~K}\right\|_{\mathrm{W}_{\infty}^{s}(E)}\right) .
\end{aligned}
$$


Similarly, the second argument in 64 can be bounded as follows:

$$
\begin{aligned}
\frac{\tau_{E}\left\|(\nabla \beta)^{\top} \mathrm{K}-\Pi_{0}^{0}\left((\nabla \beta)^{\top} \mathrm{K}\right)\right\|_{\mathrm{W}_{\infty}^{s}(E)} \leq}{\sqrt{\mathrm{K}_{E}^{V}}} & \frac{\mathcal{C}}{\sqrt{\mathrm{K}_{E}^{V}}}\left(h_{E}^{2}\left\|\frac{\mathrm{K}}{\mathrm{K}_{E}}\right\|_{\mathrm{W}_{\infty}^{s}(E)}\|\nabla \beta\|_{\mathrm{W}_{\infty}^{s}(E)}\right. \\
& \left.+h_{E}\left\|\frac{\nabla \beta}{\beta_{E}}\right\|_{\mathrm{W}_{\infty}^{s}(E)}\left\|\mathrm{K}-\Pi_{0}^{0} \mathrm{~K}\right\|_{\mathrm{W}_{\infty}^{s}(E)}\right) .
\end{aligned}
$$

Finally, the following Lemma states the continuity of $B_{\text {supg }}$, defined by 13 .

Lemma 6. Let $w \in \mathrm{H}_{k}^{1, n c}\left(\mathcal{T}_{h}\right) \cap \mathrm{H}^{2}\left(\mathcal{T}_{h}\right)$ and $v_{h} \in V_{h}$. Then,

$$
\begin{aligned}
B_{\operatorname{supg}}\left(w, v_{h}\right) \leq \mathcal{C}\left[\max _{E \in \mathcal{T}_{h}}(\right. & \left.\tau_{E}^{-\frac{1}{2}}, h_{E}^{-1} \mathcal{K}_{b, E}^{n c}\right)\|w\|+\frac{\sqrt{\mathrm{K}_{E}}}{\sqrt{\mathrm{K}_{E}^{V}}}\|w\|_{\mathrm{K} \beta \gamma}+\max _{E \in \mathcal{T}_{h}} \mathcal{K}_{b, E}^{n c}\|\nabla w\| \\
& \left.+\max _{E \in \mathcal{T}_{h}}\left(\sqrt{\tau_{E}}\|\mathrm{~K}\|_{\mathrm{W}_{\infty}^{1}(E)}\right)\left\|w-\Pi_{k-1}^{0} w\right\|_{2, E}\right]\left\|v_{h}\right\|_{\mathrm{K} \beta \gamma, E} .
\end{aligned}
$$

Proof. The proof of the continuity of $a, b$ and $c$ follows the same arguments of Lemmas (2), (3) and (4). The proof of the continuity of $d$ is slightly different, and can be done as follows:

$$
\begin{aligned}
\tau_{E}( & \left.\nabla \cdot(\mathrm{K} \nabla w), \beta \cdot \nabla v_{h}\right)_{E}=\tau_{E}\left(\nabla \cdot\left(\mathrm{K} \nabla w-\mathrm{K} \nabla \Pi_{k-1}^{0} w\right), \beta \cdot \nabla v_{h}\right)_{E} \\
& +\tau_{E}\left(\nabla \cdot\left(\mathrm{K} \nabla \Pi_{k-1}^{0} w\right), \beta \cdot \nabla v_{h}\right)_{E} \\
\leq & \tau_{E}\left\|\nabla \cdot\left(\mathrm{K} \nabla\left(w-\Pi_{k-1}^{0} w\right)\right)\right\|_{E}\left\|\beta \cdot \nabla v_{h}\right\|_{E}+\tau_{E}\left\|\nabla \cdot\left(\mathrm{K} \nabla \Pi_{k-1}^{0} w\right)\right\|_{E}\left\|\beta \cdot \nabla v_{h}\right\|_{E} \\
\leq & \left(\sqrt{\tau_{E}}\left\|(\nabla \cdot \mathrm{K}) \nabla\left(w-\Pi_{k-1}^{0} w\right)\right\|_{E}+\sqrt{\tau_{E}}\left\|\mathrm{~K} \Delta\left(w-\Pi_{k-1}^{0} w\right)\right\|_{E}\right. \\
& \left.+\left\|\sqrt{\mathrm{K}} \Pi_{k-1}^{0} \nabla w\right\|_{E}\right) \sqrt{\tau_{E}}\left\|\beta \cdot \nabla v_{h}\right\|_{E} \\
& \leq \mathcal{C}\left(\sqrt{\tau_{E}}\|\mathrm{~K}\|_{\mathrm{W}_{\infty}^{1}(E)}\left\|w-\Pi_{k-1}^{0} w\right\|_{2, E}+\frac{\sqrt{\mathrm{K}_{E}}}{\sqrt{\mathrm{K}_{E}^{V}}}\|\sqrt{\mathrm{K}} \nabla w\|_{E}\right)\|w\|_{\mathrm{K} \beta} .
\end{aligned}
$$

The above lemmas can be summarized in the following lemma, estimating the error of approximation of the exact bilinear form by the discrete bilinear form.

Lemma 7. For any given $w \in \mathrm{H}_{k}^{1, n c}\left(\mathcal{T}_{h}\right) \cap \mathrm{H}^{s+1}\left(\mathcal{T}_{h}\right)$ and any $v_{h} \in V_{h}$,

$$
\begin{aligned}
&\left|B_{\text {supg }}\left(w, v_{h}\right)-B_{\text {supg }, h}\left(w, v_{h}\right)\right| \leq \mathcal{C} \max _{E \in \mathcal{T}_{h}}\left\{\frac{\|\mathrm{K}\|_{\mathrm{W}_{\infty}^{1}(E)}}{\sqrt{\mathrm{K}_{E}}}, \sqrt{h_{E} \beta_{E}}, h_{E} \sqrt{\gamma_{E}},\right. \\
&\left.\mathcal{C}_{a, E}^{n c}, \mathcal{C}_{b, E}^{n c}, \mathcal{C}_{c, E}^{n c}, \mathcal{C}_{d, E}^{n c}, \mathcal{K}_{b, E}^{n c}\right\} h^{s}|w|_{s+1}\left\|v_{h}\right\|_{\mathrm{K} \beta},
\end{aligned}
$$

where $\mathcal{C}_{a, E}^{n c}, \mathcal{C}_{b, E}^{n c}, \mathcal{C}_{c, E}^{n c}, \mathcal{C}_{d, E}^{n c}$ and $\mathcal{K}_{b, E}^{n c}$ are defined by 46], 48), 59, ,64 and 49, 
Proof. Collecting the results of Lemmas 2, 3, 4, 5, and 6 and the approximation estimates on the polynomial projections, we get

$$
\begin{aligned}
& \mid B_{\text {supg }}\left(w, v_{h}\right)-B_{\text {supg }, h}\left(w, v_{h}\right)|\leq| B_{\text {supg }}\left(w-\Pi_{k-1}^{0} w, v_{h}\right)|+| B_{\text {supg }, h}\left(w-\Pi_{k-1}^{0} w, v_{h}\right) \mid \\
&+\left|B_{\text {supg }}\left(\Pi_{k-1}^{0} w, v_{h}\right)-B_{\text {supg }, h}\left(\Pi_{k-1}^{0} w, v_{h}\right)\right| \\
& \leq \mathcal{C}\left(\max _{E \in \mathcal{T}_{h}}\left(\frac{\sqrt{\mathrm{K}_{E}}}{\sqrt{\mathrm{K}_{E}^{\vee}}}\right)\left\|w-\Pi_{k-1}^{0} w\right\|_{\mathrm{K} \beta \gamma}\right. \\
& \quad+\max _{E \in \mathcal{T}_{h}}\left(\sqrt{\tau_{E}}\|\mathrm{~K}\|_{\mathrm{W}_{\infty}^{1}(E)}\right)\left\|w-\Pi_{k-1}^{0} w\right\|_{2, E}+\max _{E \in \mathcal{T}_{h}} \tau_{E}^{-\frac{1}{2}}\left\|w-\Pi_{k-1}^{0} w\right\| \\
&\left.\quad+\max _{E \in \mathcal{T}_{h}}\left\{\mathcal{C}_{a, E}^{n c}, \mathcal{C}_{b, E}^{n c, s+1}, \mathcal{C}_{c, E}^{n c}, \mathcal{C}_{d, E}^{n c}, \mathcal{K}_{b, E}^{n c}\right\} h^{s}|w|_{s+1}\right)\left\|v_{h}\right\|_{\mathrm{K} \beta} \\
& \leq \mathcal{C} \max _{E \in \mathcal{T}_{h}}\left\{\frac{\|\mathrm{K}\|_{\mathrm{W}_{\infty}^{1}(E)}}{\sqrt{\mathrm{K}_{E}}}, \sqrt{h_{E} \beta_{E}}, h_{E} \sqrt{\gamma_{E}}, \mathcal{C}_{a, E}^{n c}, \mathcal{C}_{b, E}^{n c, s+1}, \mathcal{C}_{c, E}^{n c}, \mathcal{C}_{d, E}^{n c}, \mathcal{K}_{b, E}^{n c}\right\} h^{s}|w|_{s+1}\left\|v_{h}\right\|_{\mathrm{K} \beta} .
\end{aligned}
$$

Due to the non-conformity of our approach and since the functions in the global virtual element space $V_{h}$ may be discontinuous, for the exact solution $u \in \mathrm{H}^{2}\left(\mathcal{T}_{h}\right) \cap \mathrm{H}_{0}^{1}(\Omega)$ and every $v_{h} \in V_{h}$ it holds that

$$
B_{\text {supg }}\left(u, v_{h}\right)=F_{\text {supg }}\left(v_{h}\right)+\mathcal{N}_{h}\left(u, v_{h}\right),
$$

where

$$
\mathcal{N}_{h}\left(u, v_{h}\right):=\sum_{E \in \mathcal{T}_{h}}\left((\mathrm{~K} \nabla u) \cdot \hat{n}-\frac{1}{2}(\beta \cdot \hat{n}) u, v_{h}\right)_{\partial E} .
$$

is called the conformity error. This term is a generalization of the one of the pure diffusion problem that is introduced and estimated in [49, Lemma 4.1].

Lemma 8 (Conformity error). Let $u \in \mathrm{H}^{s+1}\left(\mathcal{T}_{h}\right) \cap \mathrm{H}_{0}^{1}(\Omega), 1 \leq s \leq k$, be the solution of the variational problem (4). Let $\beta \in \mathrm{W}_{\infty}^{s+1}(\Omega)$ and suppose $\mathrm{K} \nabla u \in \mathrm{H}(\operatorname{div}, \Omega)$. Under the mesh regularity assumptions of Section 3.1. for every $v_{h} \in V_{h}$ it holds that

$$
\left|\mathcal{N}_{h}\left(u, v_{h}\right)\right| \leq \mathcal{C} \max _{E \in \mathcal{T}_{h}}\left\{\frac{\|\mathrm{K}\|_{\mathrm{W}_{\infty}^{s}(E)}}{\sqrt{\mathrm{K}_{E}^{V}}}, \mathcal{K}_{\mathcal{N}, E}^{n c}\right\} h^{s}\|u\|_{s+1}\left\|v_{h}\right\|_{\mathrm{K} \beta \gamma},
$$

where

$$
\mathcal{K}_{\mathcal{N}, E}^{n c}=\frac{h_{E}\|\beta \cdot \hat{n}\|_{\mathrm{W}_{\infty}^{s+\frac{1}{2}}(\partial E)}}{\sqrt{\mathrm{K}_{E}^{\vee}}} .
$$

Proof. The first term in 69 is bounded following [49, Lemma 4.1], using the fact that, 
by hypothesis, $\mathrm{K} \nabla u \cdot \hat{n}$ is continuous:

$$
\begin{aligned}
\sum_{E \in \mathcal{T}_{h}}\left((\mathrm{~K} \nabla u) \cdot \hat{n}, v_{h}\right)_{\partial E} & =\sum_{e \in \mathcal{E}_{h}}\left((\mathrm{~K} \nabla u) \cdot \hat{n}, \llbracket v_{h} \rrbracket\right)_{e}=\sum_{e \in \mathcal{E}_{h}}\left(\left(\mathrm{~K} \nabla u-\Pi_{k-1}^{0}(\mathrm{~K} \nabla u)\right) \cdot \hat{n}, \llbracket v_{h} \rrbracket\right)_{e} \\
& \leq \sum_{e \in \mathcal{E}_{h}}\left\|\left(\mathrm{~K} \nabla u-\Pi_{k-1}^{0}(\mathrm{~K} \nabla u)\right) \cdot \hat{n}\right\|_{e}\left\|\llbracket v_{h}-\Pi_{k-1}^{0, e} v_{h} \rrbracket\right\|_{e} \\
& \leq \sum_{e \in \mathcal{E}_{h}} \mathcal{C} h_{e}^{s-\frac{1}{2}}|\mathrm{~K} \nabla u|_{s, \omega_{e}} \cdot h_{e}^{\frac{1}{2}}\left\|\nabla v_{h}\right\|_{\omega_{e}} \\
& \leq \frac{\|\mathrm{K}\|_{\mathrm{W}_{\infty}^{s}(E)}}{\sqrt{\mathrm{K}_{E}^{V}}} h_{E}^{s}\|u\|_{s+1, \omega_{e}}\left\|v_{h}\right\|_{\omega_{e}} .
\end{aligned}
$$

The second term in 69 is estimated using the fact that $v_{h} \in \mathrm{H}_{k}^{1, n c}\left(\mathcal{T}_{h}\right)$. Denoting by $\Pi_{k-1}^{0, \partial E} v_{h}$ the piecewise polynomial projection of $v_{h}$ on each $e \subset \partial E$ and since $\llbracket \Pi_{k-1}^{0, e} v_{h} \rrbracket_{e}=\Pi_{k-1}^{0, e}\left(\llbracket v_{h} \rrbracket_{e}\right)=0 \forall e \subset \partial E$ because $\int_{e} \llbracket v_{h} \rrbracket q=0 \forall q \in \mathbb{P}_{k-1}(e)$, and since $(\beta \cdot \hat{n}) u$ is continuous across the edges being $\beta$ a divergence-free vector and $u \in \mathrm{H}_{0}^{1}(\Omega)$, we get

$$
\begin{aligned}
\sum_{E \in \mathcal{T}_{h}}\left((\beta \cdot \hat{n}) u, v_{h}\right)_{\partial E} & =\sum_{E \in \mathcal{T}_{h}}\left((\beta \cdot \hat{n}) u, v_{h}\right)_{\partial E}=\frac{1}{2} \sum_{E \in \mathcal{T}_{h}}\left((\beta \cdot \hat{n}) u, \llbracket v_{h} \rrbracket\right)_{\partial E} \\
& =\frac{1}{2} \sum_{E \in \mathcal{T}_{h}}\left((\beta \cdot \hat{n}) u, \llbracket v_{h}-\Pi_{k-1}^{0, \partial E} v_{h} \rrbracket\right)_{\partial E} \\
& =\frac{1}{2} \sum_{E \in \mathcal{T}_{h}}\left((\beta \cdot \hat{n}) u-\Pi_{k-1}^{0, \partial E}((\beta \cdot \hat{n}) u), \llbracket v_{h}-\Pi_{k-1}^{0, \partial E} v_{h} \rrbracket\right)_{\partial E} \\
& \leq \frac{1}{2} \sum_{E \in \mathcal{T}_{h}}\left\|(\beta \cdot \hat{n}) u-\Pi_{k-1}^{0, \partial E}((\beta \cdot \hat{n}) u)\right\|_{\partial E}\|\| v_{h}-\Pi_{k-1}^{0, \partial E} v_{h} \rrbracket \|_{\partial E} \\
& \leq \mathcal{C} \sum_{E \in \mathcal{T}_{h}} h_{E}^{s+\frac{1}{2}}|(\beta \cdot \hat{n}) u|_{s+\frac{1}{2}, \partial E} \cdot h_{E}^{\frac{1}{2}}\left\|\nabla v_{h}\right\|_{\omega_{E}} \\
& \leq \mathcal{C} \sum_{E \in \mathcal{T}_{h}} h_{E}^{s+1} \frac{\|\beta \cdot \hat{n}\|_{\mathrm{W}_{\infty}^{s+\frac{1}{2}}(\partial E)}\|u\|_{s+1, \omega_{E}}\left\|v_{h}\right\|_{\mathrm{K} \beta, \omega_{E}} .}{\sqrt{\mathrm{K}_{E}^{V}}}
\end{aligned}
$$

\subsection{Well-posedness of the discrete problem}

The following theorem proves the well-posedness of the discrete formulation.

Theorem 1 (Coercivity of $B_{\text {supg }, h}$ ). For any $v_{h} \in V_{h}$,

$$
B_{\mathrm{supg}, h}\left(v_{h}, v_{h}\right) \geq \min \left\{\frac{1}{4}, \frac{\sigma_{*}}{2}\right\} \frac{1-C_{\tau}}{2}\left\|v_{h}\right\|_{\mathrm{K} \beta \gamma},
$$

where $C_{\tau}$ is the constant introduced in (35). 
Proof. Let $v_{h} \in V_{h}$. By definition 25), it holds that

$$
b\left(v_{h}, v_{h}\right)=\frac{1}{2} \sum_{E \in \mathcal{T}_{h}}\left[\left(\beta \cdot \Pi_{k-1}^{0} \nabla v_{h}, \Pi_{k-1}^{0} v_{h}\right)_{E}-\left(\Pi_{k-1}^{0} v_{h}, \beta \cdot \Pi_{k-1}^{0} \nabla v_{h}\right)_{E}\right]=0 .
$$

Moreover, using Cauchy-Schwarz and Young inequalities we find that

$$
\begin{aligned}
\tau_{E}\left|\left(\gamma \Pi_{k-1}^{0} v_{h}, \beta \cdot \Pi_{k-1}^{0} \nabla v_{h}\right)_{E}\right| & \leq \sqrt{\gamma_{E}} \tau_{E}\left\|\sqrt{\gamma} \Pi_{k-1}^{0} v_{h}\right\|_{E}\left\|\beta \cdot \Pi_{k-1}^{0} \nabla v_{h}\right\|_{E} \\
& \leq \frac{1}{2}\left\|\sqrt{\gamma} \Pi_{k-1}^{0} v_{h}\right\|^{2}+\frac{\gamma_{E} \tau_{E}^{2}}{2}\left\|\beta \cdot \Pi_{k-1}^{0} \nabla v_{h}\right\|_{E}^{2},
\end{aligned}
$$

which implies that

$$
\tau_{E}\left(\gamma \Pi_{k-1}^{0} v_{h}, \beta \cdot \Pi_{k-1}^{0} \nabla v_{h}\right)_{E} \geq-\frac{1}{2}\left\|\sqrt{\gamma} \Pi_{k-1}^{0} v_{h}\right\|^{2}-\frac{\gamma_{E} \tau_{E}^{2}}{2}\left\|\beta \cdot \Pi_{k-1}^{0} \nabla v_{h}\right\|_{E}^{2} .
$$

Inverse inequality (34) imply that

$$
\begin{aligned}
\tau_{E}\left\|\nabla \cdot\left(\mathrm{K} \Pi_{k-1}^{0} \nabla v_{h}\right)\right\|_{E}^{2} & \leq \frac{\tilde{C}_{k}^{E} h_{E}^{2}}{\mathrm{~K}_{E}}\left\|\nabla \cdot\left(\mathrm{K} \Pi_{k-1}^{0} \nabla v_{h}\right)\right\|_{E}^{2} \leq \frac{1}{\mathrm{~K}_{E}}\left\|\mathrm{~K} \Pi_{k-1}^{0} \nabla v_{h}\right\|_{E}^{2} \\
& \leq\left\|\sqrt{\mathrm{K}} \Pi_{k-1}^{0} \nabla v_{h}\right\|_{E}^{2},
\end{aligned}
$$

since $\left\|\mathrm{K} \Pi_{k-1}^{0} \nabla v_{h}\right\|_{E} \leq \sqrt{\mathrm{K}_{E}}\left\|\sqrt{\mathrm{K}} \Pi_{k-1}^{0} \nabla v_{h}\right\|_{E}$. Using the definition of $B_{\text {supg, } h}$, cf. (23), Cauchy-Schwarz inequality, inverse inequality (34), inequalities (73), 74) and (35), we 
have

$$
\begin{aligned}
& B_{\text {supg }, h}\left(v_{h}, v_{h}\right)=\sum_{E \in \mathcal{T}_{h}}\left\{\left\|\sqrt{\mathrm{K}} \Pi_{k-1}^{0} \nabla v_{h}\right\|_{E}^{2}+\tau_{E}\left\|\beta \cdot \Pi_{k-1}^{0} \nabla v_{h}\right\|_{E}^{2}\right. \\
& +S^{E}\left(\left(I-\Pi_{k-1}^{0}\right) v_{h},\left(I-\Pi_{k-1}^{0}\right) v_{h}\right) \\
& +\left\|\sqrt{\gamma} \Pi_{k-1}^{0} v_{h}\right\|_{E}^{2}+\tau_{E}\left(\gamma \Pi_{k-1}^{0} v_{h}, \beta \cdot \Pi_{k-1}^{0} \nabla v_{h}\right)_{E} \\
& \left.-\tau_{E}\left(\nabla \cdot\left(\sqrt{\mathrm{K}} \Pi_{k-1}^{0} \nabla v_{h}\right), \beta \cdot \Pi_{k-1}^{0} \nabla v_{h}\right)_{E}\right\} \\
& \geq \sum_{E \in \mathcal{T}_{h}}\left\{\left\|\sqrt{\mathrm{K}} \Pi_{k-1}^{0} \nabla v_{h}\right\|_{E}^{2}+\left(1-\frac{\gamma_{E} \tau_{E}}{2}\right) \tau_{E}\left\|\beta \cdot \Pi_{k-1}^{0} \nabla v_{h}\right\|_{E}^{2}\right. \\
& +S^{E}\left(\left(I-\Pi_{k-1}^{0}\right) v_{h},\left(I-\Pi_{k-1}^{0}\right) v_{h}\right)+\left(1-\frac{1}{2}\right)\left\|\sqrt{\gamma} \Pi_{k-1}^{0} v_{h}\right\|_{E}^{2} \\
& \left.-\tau_{E}\left\|\nabla \cdot\left(\sqrt{\mathrm{K}} \Pi_{k-1}^{0} \nabla v_{h}\right)\right\|_{E}\left\|\beta \cdot \Pi_{k-1}^{0} \nabla v_{h}\right\|_{E}\right\} \\
& \geq \sum_{E \in \mathcal{T}_{h}}\left\{\left\|\sqrt{\mathrm{K}} \Pi_{k-1}^{0} \nabla v_{h}\right\|_{E}^{2}+\left(\frac{1}{2}-\frac{C_{\tau}}{2}\right) \tau_{E}\left\|\beta \cdot \Pi_{k-1}^{0} \nabla v_{h}\right\|_{E}^{2}\right. \\
& +S^{E}\left(\left(I-\Pi_{k-1}^{0}\right) v_{h},\left(I-\Pi_{k-1}^{0}\right) v_{h}\right)+\frac{1}{2}\left\|\sqrt{\gamma} \Pi_{k-1}^{0} v_{h}\right\|_{E}^{2} \\
& \left.-\sum_{E \in \mathcal{T}_{h}} \frac{1}{2} \tau_{E}\left\|\nabla \cdot\left(\mathrm{K} \Pi_{k-1}^{0} \nabla v_{h}\right)\right\|_{E}^{2}\right\} \\
& \geq \sum_{E \in \mathcal{T}_{h}}\left\{\frac{1}{2}\left\|\sqrt{\mathrm{K}} \Pi_{k-1}^{0} \nabla v_{h}\right\|_{E}^{2}+\left(\frac{1}{2}-\frac{C_{\tau}}{2}\right) \tau_{E}\left\|\beta \cdot \Pi_{k-1}^{0} \nabla v_{h}\right\|_{E}^{2}\right. \\
& \left.+S^{E}\left(\left(I-\Pi_{k-1}^{0}\right) v_{h},\left(I-\Pi_{k-1}^{0}\right) v_{h}\right)+\frac{1}{2}\left\|\sqrt{\gamma} \Pi_{k-1}^{0} v_{h}\right\|_{E}^{2}\right\} \\
& \geq \frac{1-C_{\tau}}{2} \sum_{E \in \mathcal{T}_{h}}\left(\left\|\sqrt{\mathrm{K}} \Pi_{k-1}^{0} \nabla v_{h}\right\|_{E}^{2}+\tau_{E}\left\|\beta \cdot \Pi_{k-1}^{0} \nabla v_{h}\right\|_{E}^{2}\right. \\
& \left.+S^{E}\left(\left(I-\Pi_{k-1}^{0}\right) v_{h},\left(I-\Pi_{k-1}^{0}\right) v_{h}\right)+\left\|\sqrt{\gamma} \Pi_{k-1}^{0} v_{h}\right\|_{E}^{2}\right) \text {. }
\end{aligned}
$$


Next, using the coercivity of the VEM stabilization in 31 we get $\forall E \in \mathcal{T}_{h}$,

$$
\begin{aligned}
\| & \sqrt{\mathrm{K}} \Pi_{k-1}^{0} \nabla v_{h}\left\|_{E}^{2}+\tau_{E}\right\| \beta \cdot \Pi_{k-1}^{0} \nabla v_{h} \|_{E}^{2}+S^{E}\left(\left(I-\Pi_{k-1}^{0}\right) v_{h},\left(I-\Pi_{k-1}^{0}\right) v_{h}\right) \\
\geq & \frac{1}{2} a_{h}\left(v_{h}, v_{h}\right)+\frac{1}{2}\left(\left\|\sqrt{\mathrm{K}} \Pi_{k-1}^{0} \nabla v_{h}\right\|_{E}^{2}+\tau_{E}\left\|\beta \cdot \Pi_{k-1}^{0} \nabla v_{h}\right\|_{E}^{2}\right. \\
& \left.+\sigma_{*}\left(\mathrm{~K}_{E}+\tau_{E} \beta_{E}^{2}\right)\left\|\nabla v_{h}-\nabla \Pi_{k-1}^{0} v_{h}\right\|_{E}^{2}\right) \\
\geq & \frac{1}{2} a_{h}\left(v_{h}, v_{h}\right)+\min \left\{\frac{1}{2}, \sigma_{*}\right\}\left(\left\|\sqrt{\mathrm{K}} \Pi_{k-1}^{0} \nabla v_{h}\right\|_{E}^{2}+\tau_{E}\left\|\beta \cdot \Pi_{k-1}^{0} \nabla v_{h}\right\|_{E}^{2}\right. \\
& \left.+\left(\mathrm{K}_{E}+\tau_{E} \beta_{E}^{2}\right)\left\|\nabla v_{h}-\Pi_{k-1}^{0} \nabla v_{h}\right\|_{E}^{2}\right) \\
\geq & \frac{1}{2} a_{h}\left(v_{h}, v_{h}\right)+\min \left\{\frac{1}{2}, \sigma_{*}\right\}\left(\left\|\sqrt{\mathrm{K}} \Pi_{k-1}^{0} \nabla v_{h}\right\|_{E}^{2}+\tau_{E}\left\|\beta \cdot \Pi_{k-1}^{0} \nabla v_{h}\right\|_{E}^{2}\right. \\
& \left.+\left\|\sqrt{\mathrm{K}}\left(\nabla v_{h}-\Pi_{k-1}^{0} \nabla v_{h}\right)\right\|_{E}^{2}+\tau_{E}\left\|\beta \cdot\left(\nabla v_{h}-\Pi_{k-1}^{0} \nabla v_{h}\right)\right\|_{E}^{2}\right) \\
\geq & \min \left\{\frac{1}{4}, \frac{\sigma_{*}}{2}\right\}\left(a_{h}\left(v_{h}, v_{h}\right)+a\left(v_{h}, v_{h}\right)\right) .
\end{aligned}
$$

In the last line we use the following inequalities:

$$
\begin{aligned}
\left\|\sqrt{\mathrm{K}} \Pi_{k-1}^{0} \nabla v_{h}\right\|_{E}^{2}+\left\|\sqrt{\mathrm{K}}\left(\nabla v_{h}-\Pi_{k-1}^{0} \nabla v_{h}\right)\right\|_{E}^{2} & \geq \frac{1}{2}\left\|\sqrt{\mathrm{K}} \nabla v_{h}\right\|_{E}^{2}, \\
\tau_{E}\left(\left\|\beta \cdot \Pi_{k-1}^{0} \nabla v_{h}\right\|_{E}^{2}+\left\|\beta \cdot\left(\nabla v_{h}-\Pi_{k-1}^{0} \nabla v_{h}\right)\right\|_{E}^{2}\right) & \geq \frac{1}{2}\left\|\beta \cdot \nabla v_{h}\right\|_{E}^{2} .
\end{aligned}
$$

\subsection{A priori error estimates}

Here, we prove the a priori error estimates showing that the stabilized formulation of the problem has optimal rates of convergence. Several constants in the error inequalities are numbered to track their dependence on the local problem coefficients.

Theorem 2. Let $u \in \mathrm{H}^{s+1}\left(\mathcal{T}_{h}\right) \cap \mathrm{H}_{0}^{1}(\Omega), 2 \leq s+1 \leq k+1$, be the solution of the variational problem (19) with $f \in \mathrm{H}^{s-1}(\Omega), \mathrm{K} \in\left[\mathrm{W}_{\infty}^{s+1}(\Omega)\right]^{d \times d}, \beta \in\left[\mathrm{W}_{\infty}^{s+1}(\Omega)\right]^{d}$ and $\gamma \in \mathrm{W}_{\infty}^{s+1}(\Omega)$. Let $u_{h} \in V_{h}$ be the solution of the VEM $(22)$ under the mesh assumption of Section 3.1. Then, for $h$ sufficiently small, it holds

$$
\begin{aligned}
\left\|u-u_{h}\right\|_{\mathrm{K} \beta \gamma} \leq \mathcal{C} h^{s} & \left\{\operatorname { m a x } _ { E \in \mathcal { T } _ { h } } \left(\frac{\|\mathrm{K}\|_{\mathrm{W}_{\infty}^{s}(E)}}{\sqrt{\mathrm{K}_{E}^{\vee}}}, \sqrt{h_{E} \beta_{E}}, h_{E} \sqrt{\gamma_{E}}, \mathcal{C}_{a, E}^{n c}, \mathcal{C}_{b, E}^{n c, s+1}, \mathcal{C}_{c, E}^{n c}, \mathcal{C}_{d, E}^{n c}, \mathcal{K}_{\mathcal{N}, E}^{n c},\right.\right. \\
\mathcal{K}_{b, E}^{n c} & )\|u\|_{s+1}+\max _{E \in \mathcal{T}_{h}} \mathcal{C}_{f, E}^{n c}\right\},
\end{aligned}
$$

where $\mathcal{C}_{a, E}^{n c}, \mathcal{C}_{b, E}^{n c, s+1}, \mathcal{C}_{c, E}^{n c}, \mathcal{C}_{d, E}^{n c}, \mathcal{K}_{\mathcal{N}, E}^{n c}$ and $\mathcal{K}_{b, E}^{n c}$ are defined by 467, 48, ,59, 64, (71) and 49 respectively, and

$$
\mathcal{C}_{f, E}^{n c}=\max \left\{\frac{\left|f-\Pi_{0}^{0} f\right|_{s-1, E}}{\sqrt{\mathrm{K}_{E}^{\vee}}}, \frac{h_{E}^{-1} \tau_{E}\left|f \beta-\Pi_{0}^{0}(f \beta)\right|_{s-1, E}}{\sqrt{\mathrm{K}_{E}^{V}}}\right\} .
$$


Proof. First, by using the triangle inequality we have

$$
\left\|u-u_{h}\right\|_{\mathrm{K} \beta \gamma} \leq\left\|u-u_{I}\right\|_{\mathrm{K} \beta \gamma}+\left\|u_{h}-u_{I}\right\|_{\mathrm{K} \beta \gamma} .
$$

The first term is bounded using 42 with $\psi=u$. We are left to estimate the norm of $e_{h}:=u_{h}-u_{I}$. Since $e_{h} \in V_{h}$, by 72 we know that

$$
\begin{aligned}
\alpha\left\|e_{h}\right\|_{\mathrm{K} \beta \gamma}^{2} \leq & B_{\text {supg }, h}\left(u_{h}-u_{I}, e_{h}\right)=F_{\text {supg }, h}\left(e_{h}\right)-B_{\text {supg }, h}\left(u_{I}, e_{h}\right) \\
= & F_{\text {supg }, h}\left(e_{h}\right)-F_{\text {supg }}\left(e_{h}\right)-\mathcal{N}_{h}\left(u, e_{h}\right)+B_{\text {supg }}\left(u, e_{h}\right)-B_{\text {supg }, h}\left(u_{I}, e_{h}\right) \\
\leq & \left|F_{\text {supg }, h}\left(e_{h}\right)-F_{\text {supg }}\left(e_{h}\right)\right|+\left|\mathcal{N}_{h}\left(u, e_{h}\right)\right|+\left|B_{\text {supg }, h}\left(u-u_{I}, e_{h}\right)\right| \\
& +\left|B_{\text {supg }}\left(u, e_{h}\right)-B_{\text {supg }, h}\left(u, e_{h}\right)\right|
\end{aligned}
$$

We estimate the first term as follows:

$$
\begin{aligned}
& \sum_{E \in \mathcal{T}_{h}}\left|\left(f, e_{h}-\Pi_{k-1}^{0} e_{h}\right)_{E}+\tau_{E}\left(f, \beta \cdot\left(\nabla e_{h}-\Pi_{k-1}^{0} \nabla e_{h}\right)\right)_{E}\right| \\
&=\sum_{E \in \mathcal{T}_{h}}\left|\left(f-\Pi_{0}^{0} f, e_{h}-\Pi_{k-1}^{0} e_{h}\right)_{E}\right|+\tau_{E}\left|\left(f \beta-\Pi_{0}^{0}(f \beta), \nabla e_{h}\right)_{E}\right| \\
&=\sum_{E \in \mathcal{T}_{h}}\left|\left(f-\Pi_{0}^{0} f-\Pi_{k-1}^{0}\left(f-\Pi_{0}^{0} f\right), e_{h}-\Pi_{k-1}^{0} e_{h}\right)_{E}\right| \\
& \quad+\tau_{E}\left|\left(f \beta-\Pi_{0}^{0}(f \beta)-\Pi_{k-1}^{0}\left(f \beta-\Pi_{0}^{0}(f \beta)\right), \nabla e_{h}\right)_{E}\right| \\
& \leq \sum_{E \in \mathcal{T}_{h}}\left\|f-\Pi_{0}^{0} f-\Pi_{k-1}^{0}\left(f-\Pi_{0}^{0} f\right)\right\|_{E}\left\|e_{h}-\Pi_{k-1}^{0} e_{h}\right\|_{E} \\
& \quad+\tau_{E}\left\|f \beta-\Pi_{0}^{0}(f \beta)-\Pi_{k-1}^{0}\left(f \beta-\Pi_{0}^{0}(f \beta)\right)\right\|_{E}\left\|\nabla e_{h}\right\|_{E} \\
& \leq \mathcal{C} h^{s} \sum_{E \in \mathcal{T}_{h}}\left(\left|f-\Pi_{0}^{0} f\right|_{s-1, E}+h_{E}^{-1} \tau_{E}\left|f \beta-\Pi_{0}^{0}(f \beta)\right|_{s-1, E}\right)\left\|\nabla e_{h}\right\|_{E} \\
& \leq \mathcal{C} h^{s} \sum_{E \in \mathcal{T}_{h}} \frac{\left|f-\Pi_{0}^{0} f\right|_{s-1, E}+h_{E}^{-1} \tau_{E}\left|f \beta-\Pi_{0}^{0}(f \beta)\right|_{s-1, E}\left\|e_{h}\right\|_{\boldsymbol{K} \beta, E} .}{\sqrt{\mathrm{K}_{E}^{V}}}
\end{aligned}
$$

Using the continuity estimate (44) to bound $a_{h},(47)$ to bound $b_{h}$, (57) to bound $c_{h}$, (62) to bound $d_{h}$, and the estimate of the VEM interpolant 42, we estimate the third term as follows:

$$
\begin{aligned}
\left|B_{\mathrm{supg}, h}\left(u-u_{I}, e_{h}\right)\right| \leq & \mathcal{C} h^{s}\left\{\left\|u-u_{I}\right\|_{\mathrm{K} \beta \gamma}\left\|e_{h}\right\|_{\mathrm{K} \beta \gamma}+\left(\max _{E \in \mathcal{T}_{h}} \tau_{E}^{-\frac{1}{2}}\left\|u-u_{I}\right\|\right.\right. \\
& \left.\left.+\max _{E \in \mathcal{T}_{h}}\left(\mathcal{C}_{b, E}^{n c, 1}+\mathcal{K}_{b, E}^{n c}\right)\left\|\nabla\left(u-u_{I}\right)\right\|\right)\left\|e_{h}\right\|_{\mathrm{K} \beta}\right\} \\
\leq & \mathcal{C} h^{s} \max _{E \in \mathcal{T}_{h}}\left\{\sqrt{\mathrm{K}_{E}}, \sqrt{h_{E} \beta_{E}}, h_{E} \sqrt{\gamma_{E}}, \mathcal{C}_{b, E}^{n c, 1}, \mathcal{K}_{b, E}^{n c}\right\}\|u\|_{s+1}\left\|e_{h}\right\|_{\mathrm{K} \beta \gamma} .
\end{aligned}
$$

The proof of 75 is concluded by using the above estimates, the estimate 70 on the non-conformity term and 68. 
Remark 9. The second argument of the max in 76 can be estimated as follows, using 43 :

$\frac{h_{E}^{-1} \tau_{E}\left|f \beta-\Pi_{0}^{0}(f \beta)\right|_{s-1, E}}{\sqrt{\mathrm{K}_{E}^{V}}} \leq \mathcal{C} \frac{\left\|\beta-\Pi_{0}^{0} \beta\right\|_{\mathrm{W}_{\infty}^{s-1}(E)}\|f\|_{s-1, E}+\|\beta\|_{\mathrm{W}_{\infty}^{s-1}(E)}\left\|f-\Pi_{0}^{0} f\right\|_{s-1, E}}{\beta_{E} \sqrt{\mathrm{K}_{E}^{V}}}$.

Remark 10. When we consider constant coefficients and a costant right-hand side all the non-consistency terms in 75 vanish, yielding the following estimate:

$$
\left\|u-u_{h}\right\|_{\mathrm{K} \beta \gamma} \leq \mathcal{C} h^{s} \max _{E \in \mathcal{T}_{h}}\left(\sqrt{\mathrm{K}_{E}}, \sqrt{h_{E} \beta_{E}}, h_{E} \sqrt{\gamma_{E}}, \mathcal{K}_{\mathcal{N}, E}^{n c}, \mathcal{K}_{b, E}^{n c}\right)\|u\|_{s+1} .
$$

Moreover, if we consider a conforming discretization, Theorem 2 proves a robust estimate with respect to the Péclet number:

$$
\left\|u-u_{h}\right\|_{\mathrm{K} \beta \gamma} \leq \mathcal{C} h^{s} \max _{E \in \mathcal{T}_{h}}\left(\sqrt{\mathrm{K}_{E}}, \sqrt{h_{E} \beta_{E}}, h_{E} \sqrt{\gamma_{E}}\right)\|u\|_{s+1},
$$

as obtained for classical Finite Elements.

\section{Numerical Results}

The numerical experiments of this section are aimed at confirming the convergence rates predicted by the a priori analysis developed in the previous sections and comparing the performance of the nonconforming VEM with that of the conforming VEM. In a preliminary stage, the consistency of the numerical method, i.e. the exactness of these methods for polynomial solutions, has been tested numerically by solving the elliptic equation with boundary and source data determined by the monomials $u(x, y)=x^{\mu} y^{\nu}$ on different set of polygonal meshes and for all possible combinations of nonnegative integers $\mu$ and $\nu$ such that $\mu+\nu \leq k$, with $k=1,2,3$. In all the cases, the error magnitude was within the arithmetic precision, thus confirming the consistency of the VEM.

To study the accuracy of the method we solve the convection-reaction-diffusion equation on the domain $\Omega=] 0,1[\times] 0,1[$. The variable coefficients of the equation are given by

$$
\begin{aligned}
& \mathrm{K}(x, y)=\alpha\left[\begin{array}{cc}
1+x^{2} & x y \\
x y & 1+y^{2}
\end{array}\right], \quad \alpha=10^{-7} \\
& \beta(x, y)=(\cos (2 \pi x), \sin (2 \pi y))^{T} \\
& \gamma(x, y)=\exp (x+y)
\end{aligned}
$$

Since the Péclet number here is in the range $\left[10^{6}, 10^{7}\right]$, all calculations are in the convection dominated regime. The forcing term and the Dirichlet boundary conditions are set such that the exact solution is

$$
u(x, y)=\sin (2 \pi x) \sin (2 \pi y)+x^{5}+y^{5}+1 .
$$



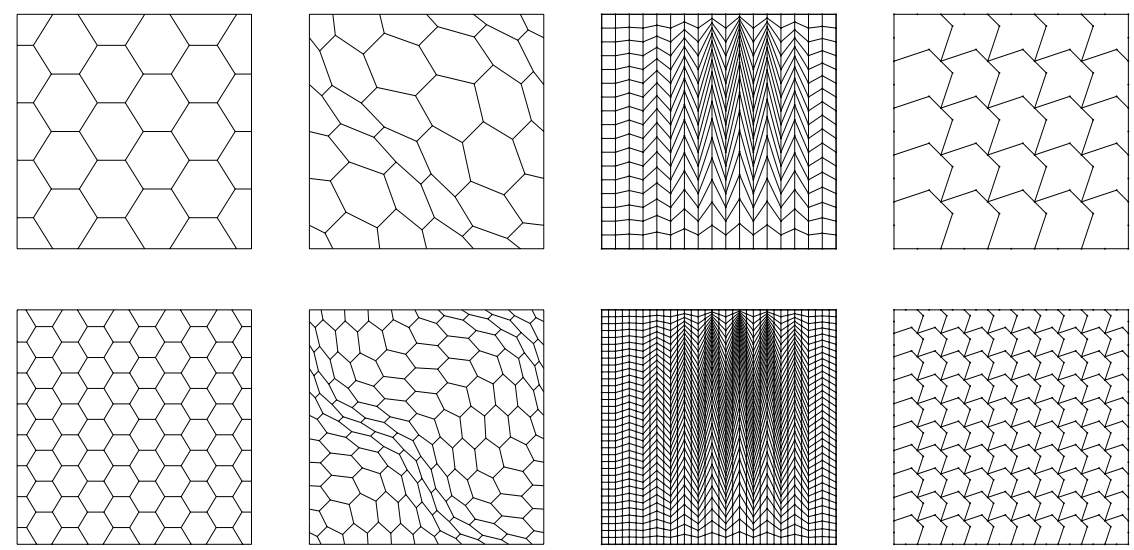

(a)

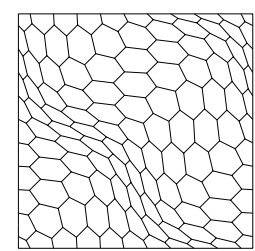

(b)

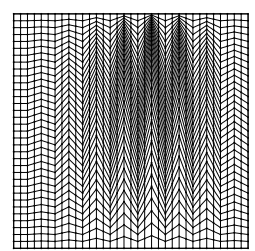

(c)

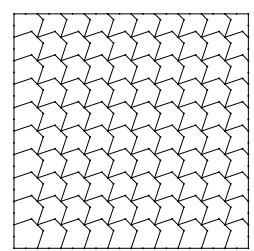

(d)

Figure 3: Base mesh (top row) and first refinement (bottom row) of the four mesh families: (a) regular hexagonal mesh; (b) remapped hexagonal mesh; (c) highly distorted quadrilateral mesh; (d) non-convex regular mesh.

The performances of the methods presented above are investigated by evaluating the rate of convergence on four different sequences of unstructured meshes, labeled by $\mathcal{M}_{1}$, $\mathcal{M}_{2}, \mathcal{M}_{3}$, and $\mathcal{M}_{4}$ respectively. The top panels of Fig. 3 show the first mesh of each sequence and the bottom panels show the mesh of the first refinement.

The meshes in $\mathcal{M}_{1}$ are built by partitioning the domain $\Omega$ into regular hexagonal cells. At the boundaries of $\Omega$ each mesh is completed by half hexagonal cells. The meshes in $\mathcal{M}_{2}$ are built as follows. First, we determine a primal mesh by remapping the position $(\widehat{x}, \widehat{y})$ of the nodes of a uniform square partition of $\Omega$ by the smooth coordinate transformation:

$$
\begin{aligned}
& x=\widehat{x}+(1 / 10) \sin (2 \pi \widehat{x}) \sin (2 \pi \widehat{y}), \\
& y=\widehat{y}+(1 / 10) \sin (2 \pi \widehat{x}) \sin (2 \pi \widehat{y}) .
\end{aligned}
$$

The corresponding mesh of $\mathcal{M}_{2}$ is built from the primal mesh by splitting each quadrilateral cell into two triangles and connecting the barycenters of adjacent triangular cells by a straight segment. The mesh construction is completed at the boundary by connecting the barycenters of the triangular cells close to the boundary to the midpoints of the boundary edges and these latters to the boundary vertices of the primal mesh. The meshes in $\mathcal{M}_{3}$ are taken from the mesh suites of the FVCA-6 Benchmark [75], and are formed by highly skewed quadrilateral cells. The meshes in $\mathcal{M}_{4}$ are obtained by filling $\Omega$ with a suitably scaled non-convex octagonal reference cell.

All the meshes are parametrised by the number of partitions in each direction. The starting mesh of every sequence is built from a $5 \times 5$ regular grid, and the refined meshes are obtained by doubling this resolution.

All errors, computed as in [51, 73, are reported in Figs. 4, 5, 6, and 7, Error values are labeled by a circle for the nonconforming VEM and by a square for the conforming VEM, that are stabilized by the method developed in 73 . Each figure shows the relative errors with respect to the maximum diameter of the discretization, in the $L^{2}$ norms (left 
panel) and in the $H^{1}$ norms (right panel). In the same figures we report the slopes $k+1$ for the $\mathrm{L}^{2}$-norm and $k$ for the $\mathrm{H}^{1}$-norm. The numerical results confirm the theoretical rate of convergence for the $\mathrm{H}^{1}$-norm. The conforming and nonconforming VEMs provide very close results on any fixed mesh, with the conforming method slightly over performing the nonconforming VEM in few cases.

To test the robustness of the approach with respect to very large Péclet numbers, we have performed some tests with values of $\mathrm{K}$ and $\beta$ in the form of $(78)$ and $(79)$, with $\alpha$ spanning a wide range of orders of magnitude $\left(\alpha \in\left\{10^{-i}: i=4, \ldots, 11\right\}\right)$, with $\gamma(x, y)=0$. In Figure 8 we display the $\mathrm{H}^{1}$ approximation error plotted with respect to the values of $\alpha$, on two of the meshes previously used. We can see that, as far as the presented tests are concerned, the error is bounded independently of the values of $\alpha$, even on non convex polygons, thus confirming the robustness of the approach.

\subsection{Approximation of internal and boundary layers}

The second test is the classic problem from 68. The computational domain and the boundary conditions are as shown in Figure 9 . The velocity forms an angle $\theta$ with the x-axis, and propagates the non-homogeneous boundary condition $u=1$ inside $\Omega$, thus generating an internal discontinuity, which is numerically approximated by an internal layer, a sharp transition between the constant solution states $u=0$ and 1 . The homogeneous boundary condition at the top of the computational domain produces a boundary layer. The diffusion coefficient is constant on $\Omega$ and given by $\mathrm{K}=10^{-6}$, while the velocity is $\beta=(\cos \theta, \sin \theta)$, and $\theta=\arctan (1)$. The Péclet number is about $10^{6}$. We solve this problem using the remapped and the regular hexagonal meshes (see plots $(a)-(b)$ of Figure 3), with resolution $40 \times 40$ (third refinement). Figures 10 and 11 show the results obtained with the conforming VEM [73] (left panels) and the nonconfoming VEM (right panels) for the polynomial degrees $k=1$ and $k=3$.

The results are quite similar to those presented in [68, 73, and are coherent with the expected behaviour of the method. Undershoots and overshoots are present near the internal layer, as is normal for this problem. However, by increasing the accuracy order of the VEM, the numerical solution becomes smoother. A thorough inspection of these plots also reveals that the nonconforming VEM tends to provide a sharper internal layer than that of the conforming VEM at the price of a relatively bigger amplitude of the spurious oscillations in the transition region.

\section{Conclusions}

In this paper, we proposed a nonconforming VEM for the advection-diffusion-reaction problem in the convection-dominated regime. Due to the strong convective field with respect to the diffusion term, we introduced the SUPG stabilization by extending to the nonconforming VEM the stabilization technique proposed in [73. The stabilization included in the virtual element formulation is a natural extension of the classical SUPG stabilization for the standard FEM. To ensure coercivity of the discrete operators, we modify the SUPG stabilization by introducing a VEM stabilization of the SUPG stabilization term. Optimal convergence rates are obtained from the convergence analysis under proper assumptions on the regularity of problem coefficients, the meshes, and the exact solution. The numerical results confirm the behaviour of the VEM that is expected 


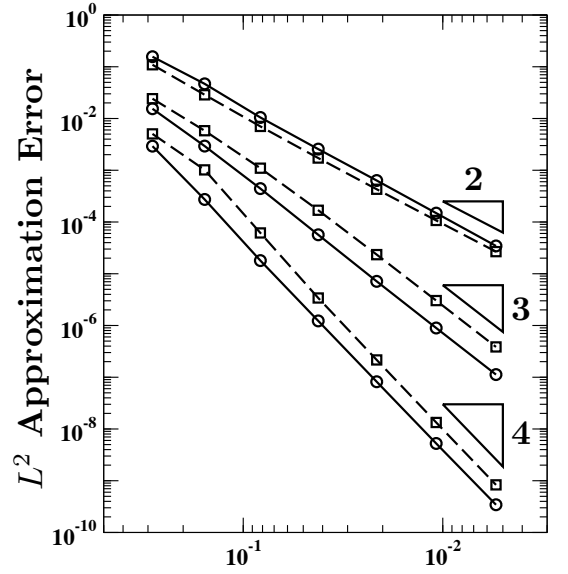

Mesh size $h$

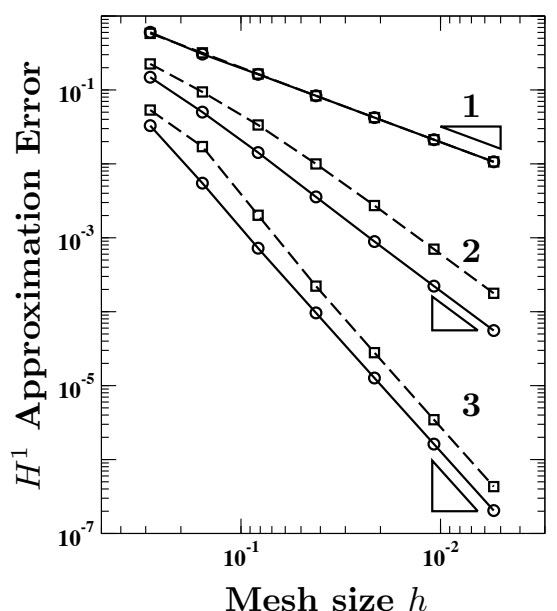

Figure 4: Relative approximation errors obtained using the conforming VEM (dashed lines labeled with squares) and the nonconforming VEM (solid lines labeled with circles) for $k=1,2,3$ (from top to bottom). Calculations are carried out using the regular hexagonal meshes of Figure 3 (a). Errors are measured in the $L^{2}$ norm (left panels) and $H^{1}$ norm (right panels), and plotted versus $h$.

from the theory and the stabilizing effect of the additional SUPG term provides stable discrete solutions even for very large Péclet numbers in the order of $10^{6}$.

\section{References}

[1] L. Beirão da Veiga, F. Brezzi, A. Cangiani, G. Manzini, L. D. Marini, A. Russo, Basic principles of virtual element methods, Mathematical Models \& Methods in Applied Sciences 23 (2013) 119-214.

[2] L. Beirão da Veiga, G. Manzini, M. Putti, Post-processing of solution and flux for the nodal mimetic finite difference method, Numerical Methods for Partial Differential Equations 31 (1) (2015) 336363.

[3] L. Beirão da Veiga, K. Lipnikov, G. Manzini, Arbitrary order nodal mimetic discretizations of elliptic problems on polygonal meshes, SIAM Journal on Numerical Analysis 49 (5) (2011) 1737-1760.

[4] F. Brezzi, A. Buffa, K. Lipnikov, Mimetic finite differences for elliptic problems, ESAIM: Mathematical Modelling and Numerical Analysis 43 (2) (2009) 277-295.

[5] G. Manzini, K. Lipnikov, J. D. Moulton, M. Shashkov, Convergence analysis of the mimetic finite difference method for elliptic problems with staggered discretizations of diffusion coefficients, SIAM Journal on Numerical Analysis 55 (6) (2017) 2956-2981. doi:10.1137/16M1108479

[6] K. Lipnikov, G. Manzini, M. Shashkov, Mimetic finite difference method, Journal of Computational Physics 257 - Part B (2014) 1163-1227, review paper.

[7] L. Beirão da Veiga, K. Lipnikov, G. Manzini, The Mimetic Finite Difference Method, I Edition, Vol. 11 of MS\&A. Modeling, Simulations and Applications, Springer, 2014.

[8] P. F. Antonietti, L. Beirão da Veiga, S. Scacchi, M. Verani, A $C^{1}$ virtual element method for the Cahn-Hilliard equation with polygonal meshes, SIAM Journal on Numerical Analysis 54 (1) (2016) $34-56$.

[9] A. Cangiani, E. H. Georgoulis, T. Pryer, O. J. Sutton, A posteriori error estimates for the virtual element method, Numerische Mathematik (2017) 1-37doi:10.1007/s00211-017-0891-9.

[10] L. Beirão da Veiga, A. Chernov, L. Mascotto, A. Russo, Basic principles of hp virtual elements on quasiuniform meshes, Mathematical Models \& Methods in Applied Sciences 26 (8) (2016) 15671598.

[11] L. Beirão da Veiga, F. Brezzi, L. D. Marini, A. Russo, H(div) and H(curl)-conforming VEM, Numerische Mathematik 133 (2) (2016) 303-332.

[12] L. Beirão da Veiga, F. Brezzi, L. D. Marini, A. Russo, Virtual element methods for general second 


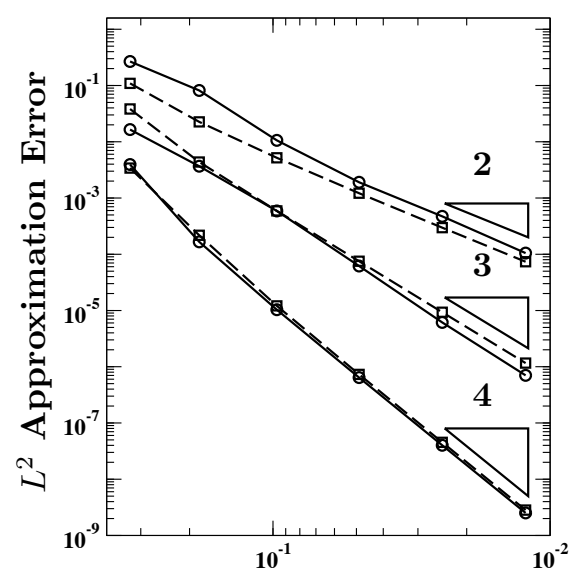

Mesh size $h$

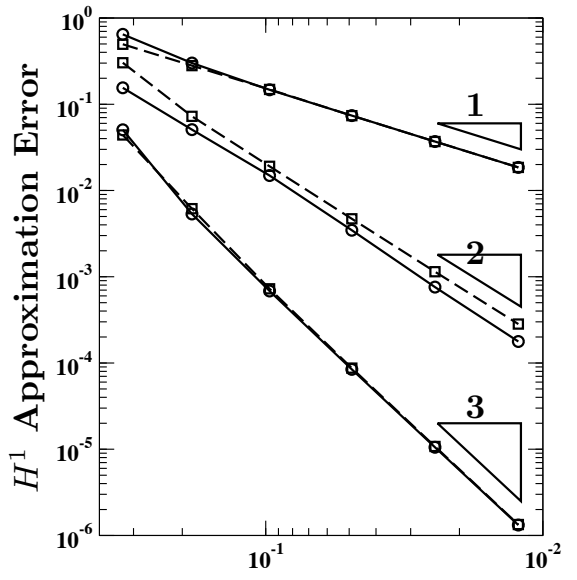

Mesh size $h$

Figure 5: Relative approximation errors obtained using the conforming VEM (dashed lines labeled with squares) and the nonconforming VEM (solid lines labeled with circles) for $k=1,2,3$ (from top to bottom). Calculations are carried out using the remapped hexagonal meshes of Figure 3 (b). Errors are measured in the $L^{2}$ norm (left panels) and $H^{1}$ norm (right panels), and plotted versus $h$.

order elliptic problems on polygonal meshes, Mathematical Models \& Methods in Applied Sciences 26 (4) (2016) 729-750.

[13] L. Beirão da Veiga, F. Brezzi, L. D. Marini, A. Russo, Mixed virtual element methods for general second order elliptic problems on polygonal meshes, ESAIM: Mathematical Modelling and Numerical Analysis 50 (3) (2016) 727-747.

[14] L. Beirão da Veiga, F. Brezzi, L. D. Marini, A. Russo, Serendipity nodal vem spaces, Computers and Fluids 141 (2016) 2-12.

[15] M. F. Benedetto, S. Berrone, A. Borio, S. Pieraccini, S. Scialò, A hybrid mortar virtual element method for discrete fracture network simulations, Journal of Computational Physics 306 (2016) 148-166. doi:10.1016/j.jcp.2015.11.034

[16] S. Berrone, A. Borio, S. Scialò, A posteriori error estimate for a PDE-constrained optimization formulation for the flow in DFNs, SIAM Journal on Numerical Analysis 54 (1) (2016) 242-261. doi:10.1137/15M1014760

[17] M. F. Benedetto, S. Berrone, S. Scialò, A globally conforming method for solving flow in discrete fracture networks using the virtual element method, Finite Elements in Analysis and Design 109 (2016) 23-36. doi:10.1016/j.finel.2015.10.003

[18] S. Berrone, S. Pieraccini, S. Scialò, Towards effective flow simulations in realistic discrete fracture networks, Journal of Computational Physics 310 (2016) 181-201. doi:10.1016/j.jcp.2016.01.009

[19] P. Wriggers, W. T. Rust, B. D. Reddy, A virtual element method for contact Computational Mechanics 58 (6) (2016) 1039-1050. doi:10.1007/s00466-016-1331-x URL http://dx.doi .org/10.1007/s00466-016-1331-x

[20] L. Beirão da Veiga, G. Manzini, Residual a posteriori error estimation for the virtual element method for elliptic problems, ESAIM: Mathematical Modelling and Numerical Analysis 49 (2015) 577-599. doi:10.1051/m2an/2014047.

[21] S. Natarajan, P. A. Bordas, E. T. Ooi, Virtual and smoothed finite elements: a connection and its application to polygonal/polyhedral finite element methods, Intenational Journal on Numerical Methods in Engineering 104 (13) (2015) 1173-1199.

[22] S. Berrone, S. Pieraccini, S. Scialò, F. Vicini, A parallel solver for large scale DFN flow simulations, SIAM Journal on Scientific Computing 37 (3) (2015) C285-C306. doi:10.1137/140984014

[23] G. Vacca, L. Beirão da Veiga, Virtual element methods for parabolic problems on polygonal meshes, Numerical Methods for Partial Differential Equations. An International Journal 31 (6) (2015) 21102134.

[24] L. Beirão da Veiga, F. Brezzi, L. D. Marini, A. Russo, The hitchhiker's guide to the virtual element method, Mathematical Models and Methods in Applied Sciences 24 (8) (2014) 1541-1573. 


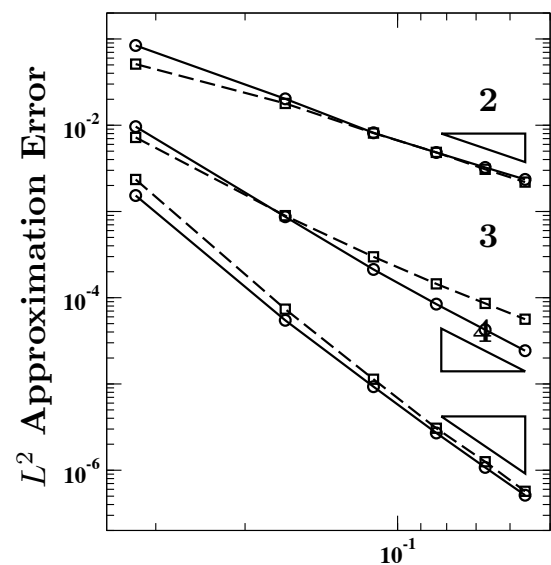

Mesh size $h$

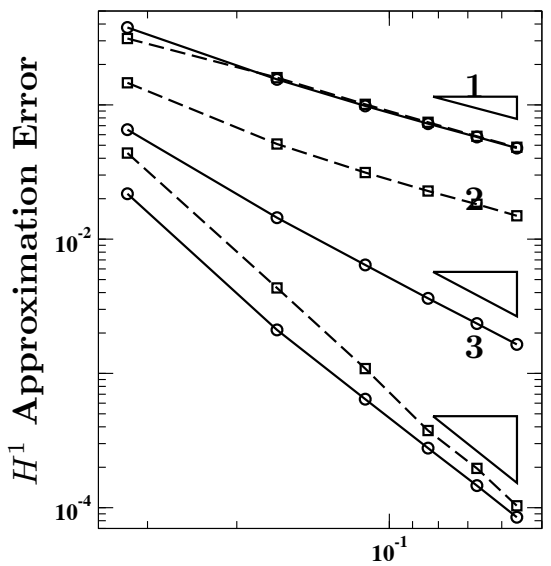

Mesh size $h$

Figure 6: Relative approximation errors obtained using the conforming VEM (dashed lines labeled with squares) and the nonconforming VEM (solid lines labeled with circles) for $k=1,2,3$ (from top to bottom). Calculations are carried out using the highly distorted quadrilateral meshes of Figure 3.c). Errors are measured in the $L^{2}$ norm (left panels) and $H^{1}$ norm (right panels), and plotted versus $h$.

[25] L. Beirão da Veiga, F. Brezzi, L. D. Marini, A. Russo, Virtual element methods for general second order elliptic problems on polygonal meshes, Mathematical Models and Methods in Applied Sciences 26 (04) (2015) 729-750. doi:10.1142/S0218202516500160

[26] L. Beirão da Veiga, L. Manzini, A virtual element method with arbitrary regularity, IMA Journal on Numerical Analysis 34 (2) (2014) 782-799, dOI: 10.1093/imanum/drt018, (first published online 2013).

[27] M. F. Benedetto, S. Berrone, S. Pieraccini, S. Scialò, The virtual element method for discrete fracture network simulations, Computer Methods in Applied Mechanics and Engineering 280 (0) (2014) 135 - 156. doi:10.1016/j.cma.2014.07.016

[28] F. Brezzi, L. D. Marini, Virtual element methods for plate bending problems, Computer Methods in Applied Mechanics and Engineering 253 (2013) 455-462. doi:10.1016/j.cma.2012.09.012

[29] M. F. Benedetto, S. Berrone, A. Borio, The Virtual Element Method for underground flow simulations in fractured media, in: Advances in Discretization Methods, Vol. 12 of SEMA SIMAI Springer Series, Springer International Publishing, Switzerland, 2016, pp. 167-186. doi:10.1007/ 978-3-319-41246-7_8

[30] S. Berrone, A. Borio, Orthogonal polynomials in badly shaped polygonal elements for the Virtual Element Method, Finite Elements in Analysis \& Design 129 (2017) 14-31. doi:10.1016/j.finel. 2017.01 .006

[31] M. F. Benedetto, S. Berrone, A. Borio, S. Pieraccini, S. Scialo, The virtual element method for discrete fracture network flow and transport simulations, in: ECCOMAS Congress 2016 - Proceedings of the 7th European Congress on Computational Methods in Applied Sciences and Engineering, Vol. 2, 2016, pp. 2953-2970.

[32] E. Wachspress, A rational finite element basis, Vol. 114 of Mathematics in Science and Engineering, Academic Press, Inc., New York-London, 1975

[33] N. Sukumar, A. Tabarraei, Conforming polygonal finite elements, International Journal for Numerical Methods in Engineering 61 (12) (2004) 2045-2066.

[34] C. Hofreither, U. Langer, S. Weißer, Convection-adapted BEM-based FEM, ZAMM-Journal of Applied Mathematics and Mechanics/Zeitschrift für Angewandte Mathematik und Mechanik 96 (12) (2016) 1467-1481.

[35] S. Weißer, Residual error estimate for bem-based fem on polygonal meshes, Numerische Mathematik 118 (4) (2011) 765-788.

[36] J. Droniou, Finite volume schemes for diffusion equations: Introduction to and review of modern methods, Mathematical Models and Methods in Applied Sciences 24 (08) (2014) 1575-1619.

[37] J. Droniou, R. Eymard, T. Gallouët, R. Herbin, A unified approach to mimetic finite difference, 


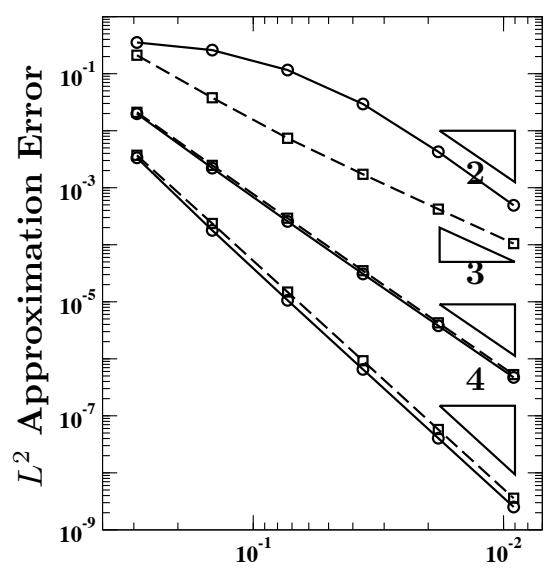

Mesh size $h$

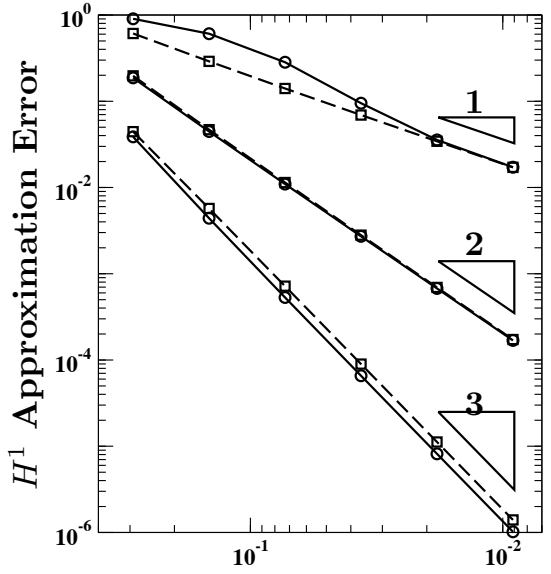

Mesh size $h$

Figure 7: Relative approximation errors obtained using the conforming VEM (dashed lines labeled with squares) and the nonconforming VEM (solid lines labeled with circles) for $k=1,2,3$ (from top to bottom). Calculations are carried out using the non convex meshes of Figure 3(d). Errors are measured in the $L^{2}$ norm (left panels) and $H^{1}$ norm (right panels), and plotted versus $h$.

hybrid finite volume and mixed finite volume methods, Mathematical Models and Methods in Applied Sciences 20 (02) (2010) 265-295. doi:10.1142/S0218202510004222

[38] D. A. D. Pietro, A. Ern, Hybrid high-order methods for variable-diffusion problems on general meshes, Comptes Rendus Mathematique 353 (1) (2015) 31-34. doi:10.1016/j.crma.2014.10.013

[39] D. A. Di Pietro, A. Ern, Mathematical aspects of discontinuous Galerkin methods, Springer Science \& Business Media, 2011.

[40] A. Cangiani, E. H. Georgoulis, P. Houston, $h p$-version discontinuous Galerkin methods on polygonal and polyhedral meshes, Mathematical Models and Methods in Applied Sciences 24 (10) (2014) 2009-2041.

[41] B. Cockburn, J. Gopalakrishnan, R. Lazarov, Unified hybridization of discontinuous Galerkin, mixed, and continuous Galerkin methods for second order elliptic problems,, SIAM Journal on Numerical Analysis 47 (2) (2009) 1319-1365.

[42] J. Droniou, R. Eymard, R. Herbin, Gradient schemes: Generic tools for the numerical analysis of diffusion equations, ESAIM: Mathematical Modelling and Numerical Analysis 50 (3) (2016) 749 781. doi:10.1051/m2an/2015079

[43] J. Droniou, R. Eymard, R. Herbin, Gradient schemes: a generic framework for the discretisation of linear, nonlinear and nonlocal elliptic and parabolic problems, Mathematical Models and Methods in Applied Sciences 23 (13) (2013) 2395-2432. doi:10.1142/S0218202513500358

[44] G. Manzini, A. Russo, N. Sukumar, New perspectives on polygonal and polyhedral finite element methods, Mathematical Models \& Methods in Applied Sciences 24 (8) (2014) 1621-1663.

[45] A. Cangiani, G. Manzini, A. Russo, N. Sukumar, Hourglass stabilization of the virtual element method, International Journal on Numerical Methods in Engineering 102 (3-4) (2015) 404-436.

[46] D. A. Di Pietro, J. Droniou, G. Manzini, Discontinuous skeletal gradient discretisation methods on polytopal meshes, Journal of Computational Physics 355 (2018) 397-425. doi:https://doi.org/ $10.1016 / j \cdot j c p .2017 .11 .018$

[47] A. Cangiani, V. Gyya, G. Manzini, S. O., Chapter 14: Virtual element methods for elliptic problems on polygonal meshes, in: K. Hormann, N. Sukumar (Eds.), Generalized Barycentric Coordinates in Computer Graphics and Computational Mechanics, CRC Press, Taylor \& Francis Group, 2017, pp. $1-20$.

[48] B. Ahmad, A. Alsaedi, F. Brezzi, L. D. Marini, A. Russo, Equivalent projectors for virtual element methods, Computers \& Mathematics with Applications 66 (2013) 376-391.

[49] B. Ayuso de Dios, K. Lipnikov, G. Manzini, The non-conforming virtual element method, ESAIM: Mathematical Modelling and Numerical Analysis 50 (3) (2016) 879-904.

[50] K. Lipnikov, G. Manzini, A high-order mimetic method for unstructured polyhedral meshes, Journal 
Conforming VEM

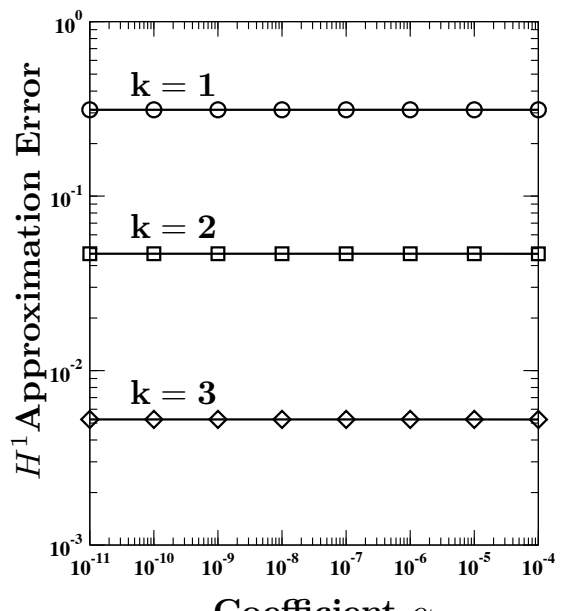

Coefficient $\alpha$

\section{Nonconforming VEM}

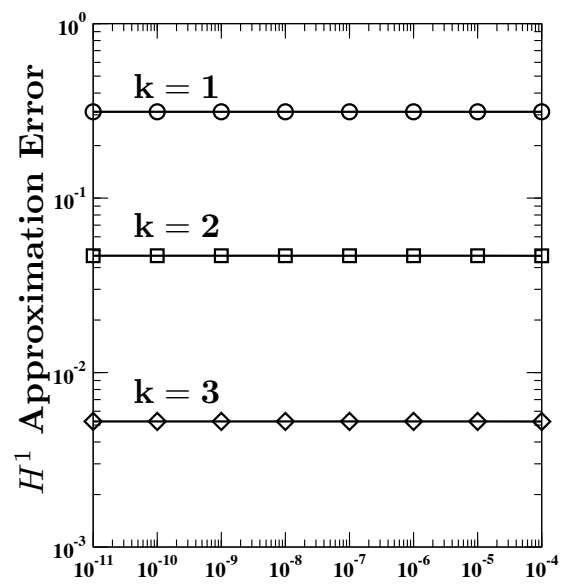

Coefficient $\alpha$

\section{Mesh of regular hexagons (a)}

\section{Conforming VEM}

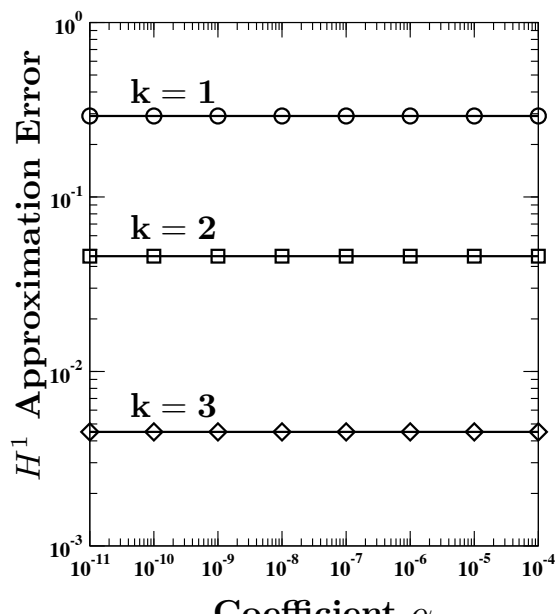

Nonconforming VEM

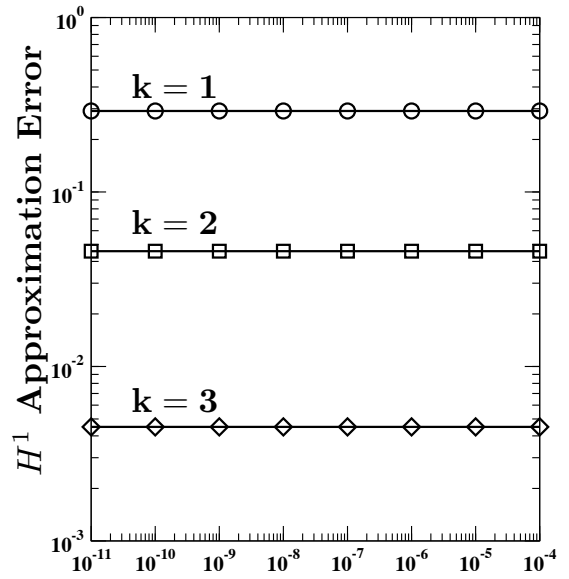

Coefficient $\alpha$

\section{Mesh of regular non convex cells (d)}

Figure 8: $H^{1}$ relative approximation error versus the viscous coefficient $\alpha \in\left[10^{-11}, 10^{-4}\right]$ using the first refined mesh of mesh families $(a)$ (top panels) and $(d)$ (bottom panels) for the test case with $\gamma(x, y)=0$. The problem is solved by applying the conforming VEM (left panel) and nonconforming VEM (right panel) of degree $k=1$ (circles), $k=2$ (squares), $k=3$ (diamonds). 


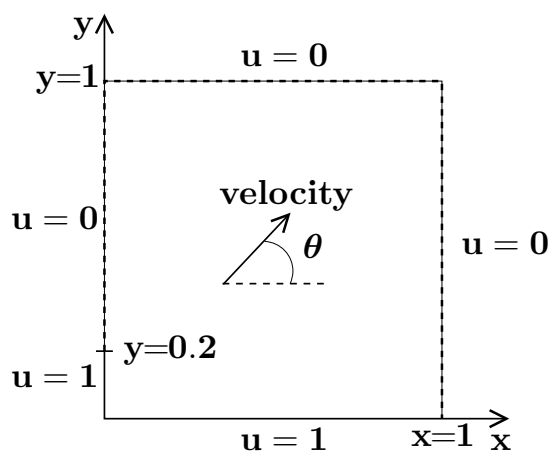

Figure 9: Test 2: domain and boundary conditions.

of Computational Physics 272 (2014) 360-385.

[51] A. Cangiani, G. Manzini, O. Sutton, Conforming and nonconforming virtual element methods for elliptic problems, IMA Journal on Numerical AnalysisAccepted June 2016. doi:10.1093/imanum/ drw036

[52] A. Cangiani, V. Gyrya, G. Manzini, The non-conforming virtual element method for the Stokes equations, SIAM Journal on Numerical Analysis 54 (6) (2016) 3411-3435.

[53] P. F. Antonietti, G. Manzini, M. Verani, The fully nonconforming Virtual Element method for biharmonic problems, M3AS Math. Models Methods Appl. Sci. 28 (2).

[54] J. Zhao, S. Chen, B. Zhang, The nonconforming virtual element method for plate bending problems, Mathematical Models \& Methods in Applied Sciences 26 (9) (2016) 1671-1687.

[55] M. Crouzeix, P.-A. Raviart, Conforming and nonconforming finite element methods for solving the stationary Stokes equations. I, Rev. Francaise Automat. Informat. Recherche Opérationnelle Sér. Rouge 7 (R-3) (1973) 33-75.

[56] M. Fortin, M. Soulie, A non-conforming piecewise quadratic finite element on triangles, International Journal for Numerical Methods in Engineering 19 (4) (1983) 505-520. doi:10.1002/nme. 1620190405

URL http://dx.doi .org/10.1002/nme.1620190405

[57] M. Crouzeix, R. S. Falk, Nonconforming finite elements for the Stokes problem, Math. Comp. 52 (1989) 437-456.

[58] G. Stoyan, A. E. Baran, Crouzeix-Velte decompositions for higher-order finite elements Comput. Math. Appl. 51 (6-7) (2006) 967-986. doi:10.1016/j.camwa.2005.10.011

URL http://dx.doi.org/10.1016/j.camwa. 2005.10.011

[59] A. E. Baran, G. Stoyan, Gauss-Legendre elements: A stable, higher order non-conforming finite element family, Computing 79 (1) (2007) 1-21. doi:10.1007/s00607-007-0219-1 URL http://dx.doi.org/10.1007/s00607-007-0219-1

[60] M. Ainsworth, Robust a posteriori error estimation for nonconforming finite element approximation, SIAM Journal on Numerical Analysis 42 (6) (2005) 2320-2341. doi:10.1137/S0036142903425112

[61] R. Rannacher, S. Turek, Simple nonconforming quadrilateral Stokes element, Numer. Methods Partial Differential Equations 8 (2) (1992) 97-111. doi:10.1002/num.1690080202

[62] G. Matthies, L. Tobiska, Inf-sup stable non-conforming finite elements of arbitrary order on triangles. Numerische Mathematik 102 (2) (2005) 293-309. doi:10.1007/s00211-005-0648-8 URL http://dx.doi.org/10.1007/s00211-005-0648-8

[63] G. Matthies, Inf-sup stable nonconforming finite elements of higher order on quadrilaterals and hexahedra, ESAIM: Mathematical Modelling and Numerical Analysis 41 (5) (2007) 855-874. URL http://eudml.org/doc/250052

[64] S. Ganesan, L. Tobiska, Stabilization by local projection for convection-diffusion and incompressible flow problems, Journal of Scientific Computing 43 (3) (2010) 326-342.

[65] F. Brezzi, L. P. Franca, A. Russo, Further considerations on residual-free bubbles for advectivediffusive equations, Computer Methods in Applied Mechanics and Engineering 166 (1-2) (1998) $25-33$.

[66] L. P. Franca, L. Tobiska, Stability of the residual free bubble method for bilinear finite elements on rectangular grids, IMA Journal of Numerical Analysis 22 (1) (2002) 73-87. 

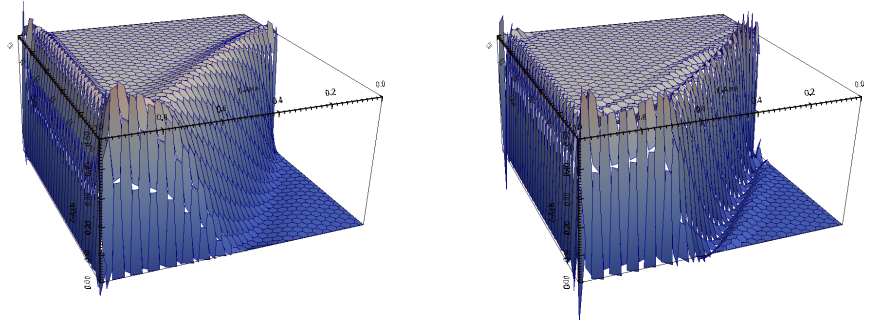

$\mathrm{k}=1$
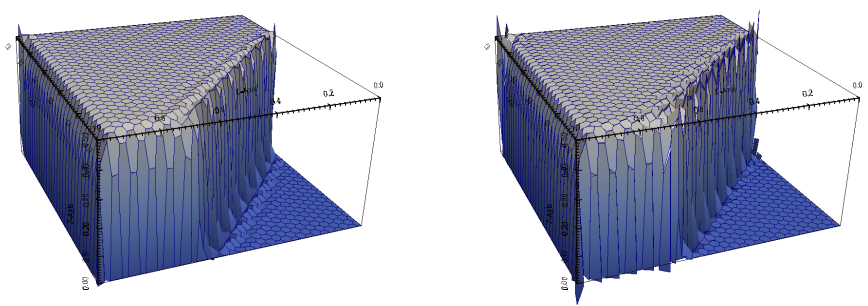

$$
\mathrm{k}=\mathbf{3}
$$

Figure 10: Test Case 2: conforming VEM (left panels) and nonconforming VEM (right panels) for $k=1$ and $k=3$ and using a regular hexagonal mesh (third refinement).
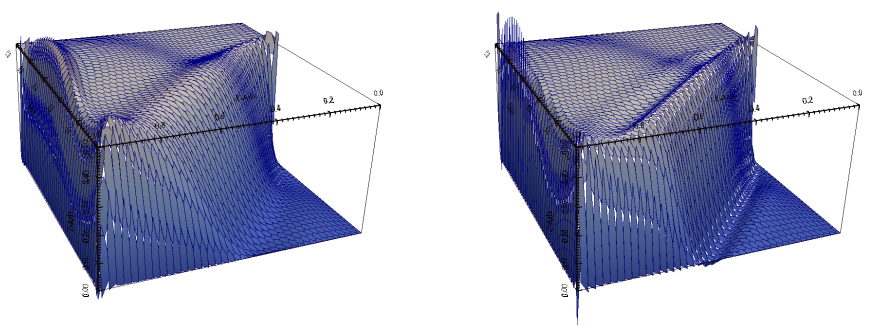

$$
\mathrm{k}=1
$$
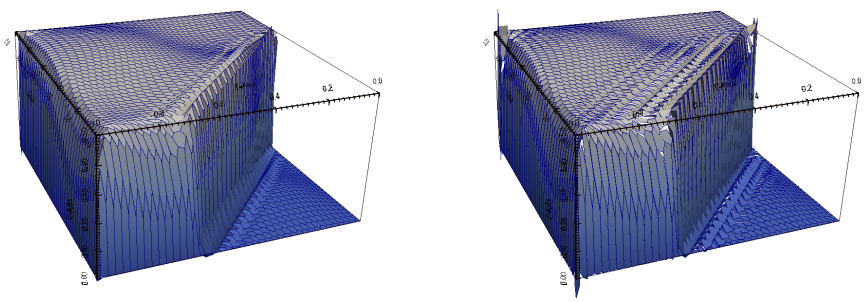

$$
\mathrm{k}=3
$$

Figure 11: Test Case 2: conforming VEM (left panels) and nonconforming VEM (right panels) for $k=1$ and $k=3$ and using a remapped hexagonal mesh (third refinement). 
[67] A. N. Brooks, T. J. Hughes, Streamline upwind/Petrov-Galerkin formulations for convection dominated flows with particular emphasis on the incompressible Navier-Stokes equations, Computer Methods in Applied Mechanics and Engineering 32 (1-3) (1982) 199-259.

[68] L. P. Franca, S. L. Frey, T. Hughes, Stabilized finite element methods: I. Application to the advective-diffusive model, Computer Methods in Applied Mechanics and Engineering 95 (2) (1992) $253-276$.

[69] T. Gelhard, G. Lube, M. A. Olshanskii, J.-H. Starcke, Stabilized finite element schemes with LBB-stable elements for incompressible flows, Journal of Computational and Applied Mathematics 177 (2) (2005) 243-267.

[70] H.-G. Roos, M. Stynes, L. Tobiska, Robust numerical methods for singularly perturbed differential equations: convection-diffusion-reaction and flow problems, Springer Science \& Business Media, 2008.

[71] E. Burman, G. Smith, Analysis of the space semi-discretized SUPG method for transient convectiondiffusion equations, Mathematical Models and Methods in Applied Sciences 21 (10) (2011) 20492068.

[72] E. Burman, Consistent SUPG-method for transient transport problems: Stability and convergence, Computer Methods in Applied Mechanics and Engineering 199 (17) (2010) 1114-1123.

[73] M. F. Benedetto, S. Berrone, A. Borio, S. Pieraccini, S. Scialò, Order preserving SUPG stabilization for the virtual element formulation of advection-diffusion problems, Computer Methods in Applied Mechanics and Engineering 311 (2016) 18-40.

[74] L. Beirão da Veiga, F. Brezzi, L. D. Marini, A. Russo, Virtual element methods for general second order elliptic problems on polygonal meshes, Mathematical Models and Methods in Applied Sciences 26 (04) (2015) 729-750. doi:10.1142/S0218202516500160

[75] R. Eymard, G. Henri, R. Herbin, R. Klofkorn, G. Manzini, 3D Benchmark on discretizations schemes for anisotropic diffusion problems on general grids, in: J. Fort, J. Furst, J. Halama, R. Herbin, F. Hubert (Eds.), Finite Volumes for Complex Applications VI. Problems \& Perspectives, Vol. 2, Springer, Prague, 2011, pp. 95-130. 